\title{
Tailoring and Understanding the Mechanical Properties of Nanoparticle-Shelled Bubbles
}

\author{
Teresa Brugarolas, $^{\dagger}$ Daniel S. Gianola, ${ }^{*,+, \S}$ Lei Zhang, ${ }^{\dagger, \|}$ Gregory M. Campbell, ${ }^{\perp}$ John L. Bassani, ${ }^{*, \S}$ \\ Gang Feng, ${ }^{*, \perp}$ and Daeyeon Lee*,†
}

${ }^{\dagger}$ Department of Chemical and Biomolecular Engineering, ${ }^{\star}$ Department of Materials Science and Engineering, and ${ }^{\S}$ Department of Mechanical Engineering and Applied Mechanics, University of Pennsylvania, Philadelphia, Pennsylvania 19104, United States,

"Department of Mechanical Engineering, University of Alaska Fairbanks, Fairbanks, Alaska 99775, United States

${ }^{\perp}$ Department of Mechanical Engineering, Villanova University, Villanova, Pennsylvania 19085, United States

\section{Supporting Information}

ABSTRACT: One common approach to generate lightweight materials with high specific strength and stiffness is the incorporation of stiff hollow microparticles (also known as bubbles or microballoons) into a polymeric matrix. The mechanical properties of these composites, also known as syntactic foams, greatly depend on those of the hollow microparticles. It is critical to precisely control the properties of these bubbles to fabricate lightweight materials that are suitable for specific applications. In this paper, we present a

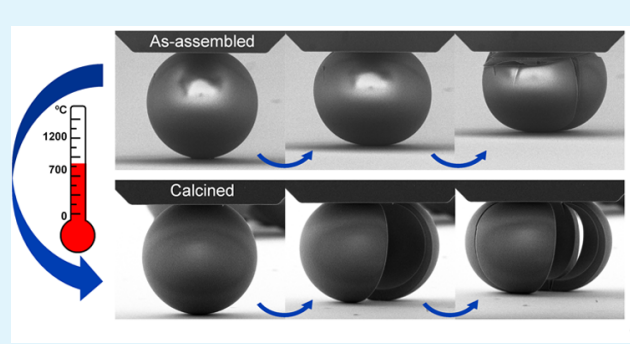

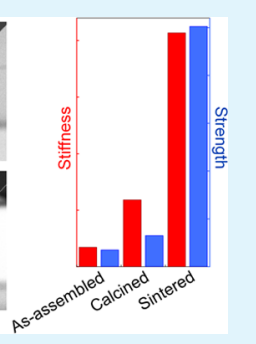
method to tailor the mechanical properties and response of highly monodisperse nanoparticle-shelled bubbles using thermal treatment. We characterize the mechanical properties of individual as-assembled bubbles as well as those of thermally treated ones using nanoindentation and quantitative in situ compression tests. As-assembled bubbles display inelastic response, whereas thermally treated bubbles behave elastically. We also show that the stiffness and strength of bubbles are enhanced significantly, as much as 12 and 14 times that of the as-assembled bubbles, respectively, via thermal treatment. We complement the experimental results with finite element analysis (FEA) to understand the effect of shell thickness nonuniformity as well as the inelasticity on the mechanical response and fracture behavior of these bubbles. We demonstrate that the failure mechanism of bubbles incorporated into a polymer composite depends on the structure of the bubbles.

KEYWORDS: nanoparticle-shelled bubbles, calcination, sintering, mechanical testing, lightweight materials

\section{INTRODUCTION}

Light-weight materials enhance the energy efficiency of vehicles and equipment used in transportation, aerospace, and construction industries; ${ }^{1-4}$ however, finding a low-density material that possesses a desirable set of mechanical properties is challenging because the stiffness and strength of materials are generally proportional to their density. ${ }^{5-8}$ One approach that addresses this limitation is the fabrication of composites made of polymer and strong hollow particles, also known as bubbles. ${ }^{9,10}$ The presence of these bubbles made of an inorganic material, such as glass, decreases the density of the composite and at the same time increases its specific strength. In addition, the incorporation of bubbles can potentially impart unique thermal, optical, and acoustic properties to the composites. $^{11-16}$

Needless to say, the mechanical properties of individual bubbles greatly affect the properties of these composites, also known as syntactic foams. ${ }^{17-19}$ It is highly desirable, therefore, to control the mechanical response of bubbles to suit the specific requirements of the final application. For example, high strength and lightweight or high buoyancy are required in composite materials used for aircraft structures and underwater modules such as deep-sea exploration vehicles. ${ }^{4,20}$ In contrast, hollow particles with high deformability would be useful in generating flexible portable devices, ${ }^{21}$ noise control structures, ${ }^{22}$ impact absorbers, ${ }^{23}$ and sports equipment. ${ }^{24}$ In addition to achieving desirable mechanical properties, high uniformity in the size and properties of these hollow particles could enable accurate prediction of the structure-property relationship of the composites containing bubbles. ${ }^{25}$ Unfortunately, conventional methods of bubble preparation result in particles with large heterogeneity in size and properties. ${ }^{26,27}$ It is also challenging to tailor the mechanical response and to systematically characterize individual bubbles. ${ }^{28,29}$ More importantly, even a small number of very weak (i.e., defective) bubbles could result in mechanically fragile regions within the final composites, seriously jeopardizing the reliability of these hollow

Received: April 15, 2014

Accepted: June 23, 2014 


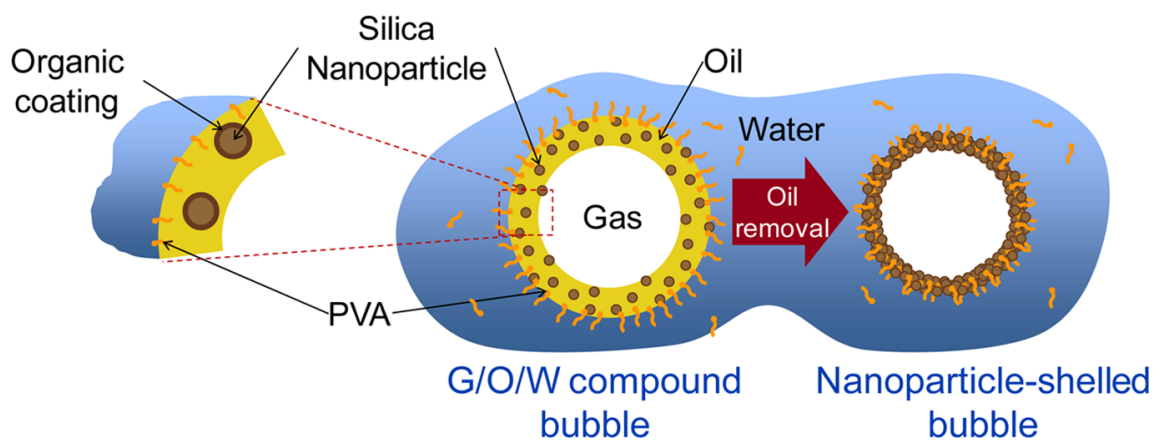

Figure 1. Schematic illustration of nanoparticle-shelled bubble formation from a gas-in-oil-in-water $(\mathrm{G} / \mathrm{O} / \mathrm{W})$ compound bubble generated with a microfluidic technique. The removal of the toluene in the oil layer drives jamming and compaction of the suspended silica nanoparticles, which forms a solid shell around the inner gas phase.

a)

\section{Dried Nanoparticle- Shelled Bubble}

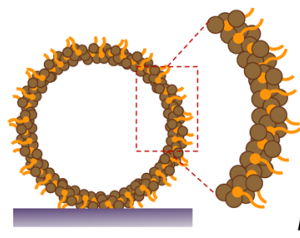

Removal of organics renders shell porous

Calcined Shelled

Bubble

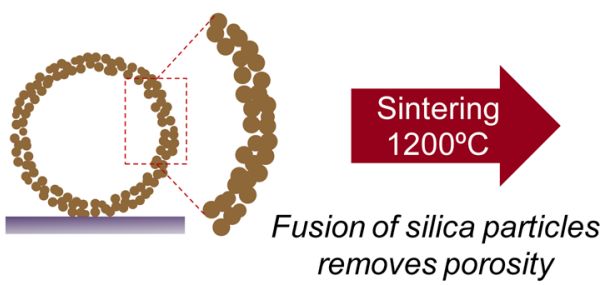

Sintered Shelled

Bubble

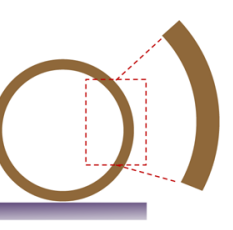

b)
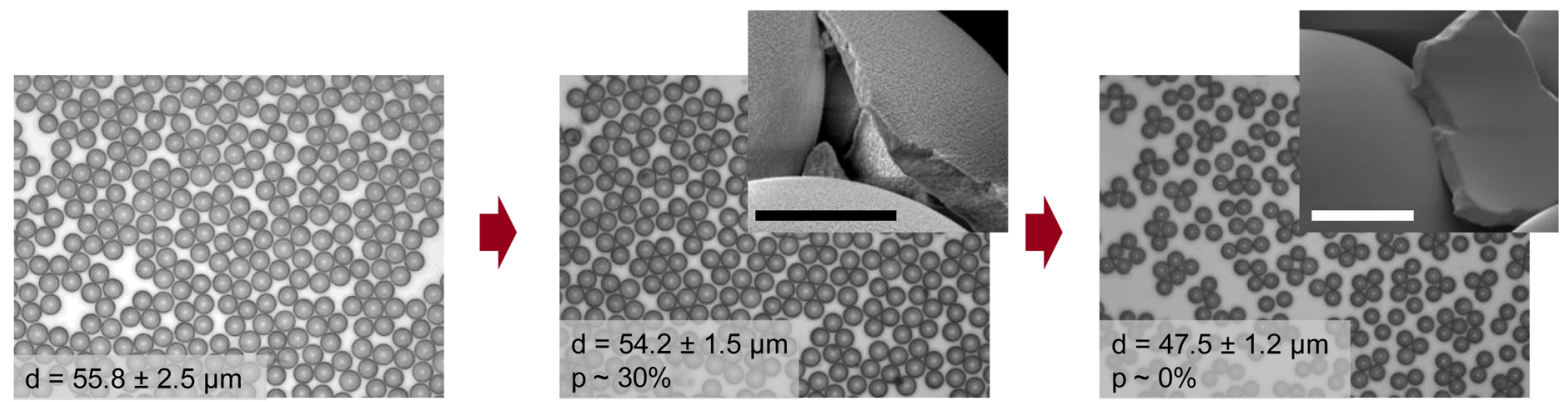

Figure 2. (a) Schematic representation of physical modification of nanoparticle-shelled bubbles with thermal treatment and (b) optical micrographs (inset: SEM micrographs) of nanoparticle-shelled bubbles on a substrate after heat treatment. $d$ and $p$ represent the outer diameter of the bubbles and the porosity of the bubble shells, respectively. Inset scale bars $=10 \mu \mathrm{m}$.

particles as fillers to generate mechanically robust composites for applications involving sustained stresses. ${ }^{30}$

In this work, we use a microfluidic technique to generate highly monodisperse nanoparticle-shelled bubbles ${ }^{31-33}$ and demonstrate that their mechanical properties can be tailored using thermal treatment. The mechanical response of these bubbles is studied experimentally using quantitative ex situ and in situ characterization methods and computationally using finite element analysis (FEA). We show that thermal treatment significantly enhances the strength of nanoparticle-shelled bubbles and also changes the deformation mode of the bubbles under load. Our finite element analysis shows that the geometry as well as the inelasticity of the bubble shells can significantly affect their mechanical response. Furthermore, we show that the failure mode of these bubbles in a polymer-bubble composite depends strongly on the structure of the individual bubbles.

\section{RESULTS AND DISCUSSION}

Generation and Thermal Treatment of Monodisperse Nanoparticle-Shelled Bubbles. Nanoparticle-shelled bubbles are generated using microfluidic gas-in-oil-in-water $(\mathrm{G} / \mathrm{O} /$
W) compound bubbles as templates. ${ }^{31,32,34-44}$ The three immiscible fluid phases used for the generation of $\mathrm{G} / \mathrm{O} / \mathrm{W}$ compound bubbles in a glass capillary microfluidic device are nitrogen, hydrophobic silica nanoparticles suspended in toluene, and an aqueous solution containing poly(vinyl alcohol) (PVA) forming the inner, middle, and outer phases of the compound bubble, respectively. As described in our previous reports, this microfluidic approach permits the preparation of monodisperse compound bubbles at a high rate with precise control over diameter and shell thickness by controlling the geometry of the microfluidic device, the physical properties (viscosity, density, surface tension etc.), and the flow rates of the three fluids. ${ }^{31,33}$ PVA in the outer phase stabilizes the oilwater interface during the microfluidic formation of the compound bubbles, preventing their coalescence and rupture. $^{31,45}$ After the generation of $\mathrm{G} / \mathrm{O} / \mathrm{W}$ compound bubbles, the toluene from the middle phase is allowed to evaporate at room temperature, causing the silica nanoparticles to jam around the gas bubble. The evaporation of toluene transforms the middle layer from a fluid phase to a water-impermeable solid phase made of jammed nanoparticles, as depicted in Figure 1. This thin shell formed by the compaction of silica nanoparticles in the middle phase imparts long-term stability to 
a)

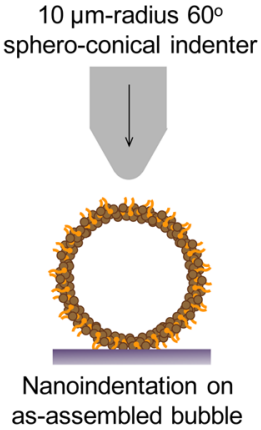

c)

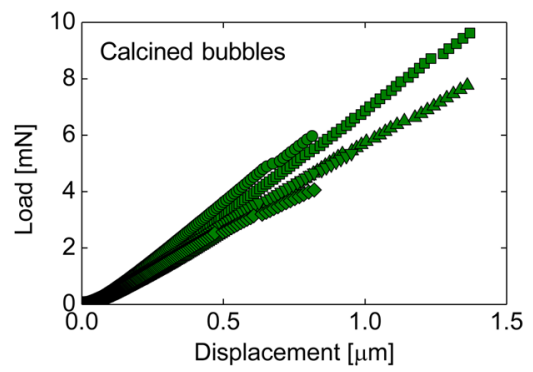

b)

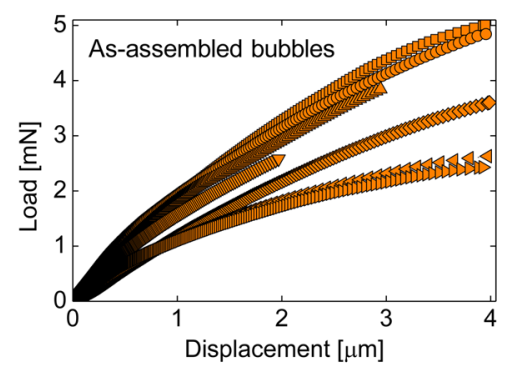

d)

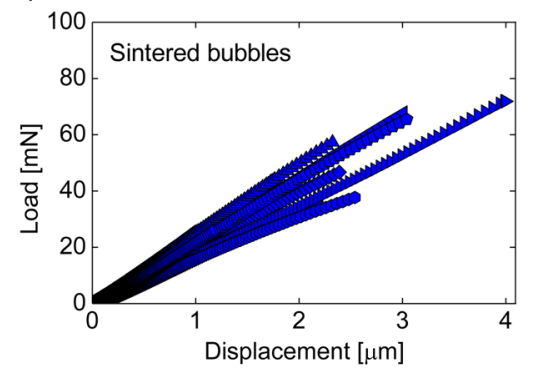

Figure 3. (a) Schematic illustration of nanoindentation on a single bubble with a spheroconical indenter. Load-displacement results from ex situ nanoindentation tests performed on (b) seven as-assembled bubbles, (c) five calcined bubbles, and (d) nine sintered bubbles.

the bubbles. ${ }^{31}$ The shell comprises randomly packed silica nanoparticles and residual PVA remaining on the shell surface. It is important to note that the silica nanoparticles have an organic layer that renders them hydrophobic and colloidally stable in toluene. This organic coating on the silica nanoparticles remains in the shell after the evaporation of toluene from the oil phase of the compound bubble.

Nanoparticle-shelled bubbles are dried on top of a silicon substrate forming a monolayer of bubbles. Our previous study showed that it is important to keep the ratio of shell thickness to bubble radius above a critical value $(0.042)$ to keep these bubbles from collapsing during water evaporation. ${ }^{32}$ In this work, we generate nanoparticle-shelled bubbles that have an initial diameter of $40-60 \mu \mathrm{m}$ and an average shell thickness of 2-3 $\mu \mathrm{m}$. The dried bubbles on the Si wafer are further modified by thermal treatment, as shown in Figure $2 a^{46-48}$ Bubbles are calcined at $700{ }^{\circ} \mathrm{C}$, which completely removes all organic components from their shells, as confirmed by thermogravimetric analysis (TGA; see Figure S1 of the Supporting Information). ${ }^{49,50}$ The removal of the organic components renders the shell porous and water-permeable (see Figure S2 of the Supporting Information). The diameter of the bubbles slightly decreases due to the elimination of the organics and the partial fusion of silica nanoparticles upon calcination. The geometry of the calcined bubbles can be modified by using a different geometry of as-assembled bubbles. The calcination process, however, does not compromise the sphericity or integrity of the bubbles. The bubble outer surface remains rough and porous, as shown by the SEM micrograph in the inset of Figure 2b.

The bubbles can be further modified by subjecting them to a $1200{ }^{\circ} \mathrm{C}$ thermal treatment. At this temperature, silica nanoparticles in the shell completely sinter to form a nonporous solid silica structure. Although this temperature is well below the melting temperature of bulk $\mathrm{SiO}_{2}\left(\sim 1600{ }^{\circ} \mathrm{C}\right)$, the use of nanoparticles enhances the processability of these bubbles by lowering the sintering temperature significantly. The shell, as can be seen in the inset of Figure $2 b$, becomes smooth, indicating that it has lost its porosity. The diameter of the bubble and the shell thickness decrease significantly from their original values upon sintering; however, the bubbles maintain their spherical geometry. The diameter and thickness of the sintered bubbles can be modified by using as-assembled bubbles with different geometry for sintering. The sintering process partially fuses some bubbles with each other or with the substrate, especially if the sintering time is extended beyond 3 h. However, due to the high strength of the sintered shells, the bubbles can be readily separated and redispersed with little damage using brief sonication $(<1 \mathrm{~s})$. These bubbles float when redispersed in water, indicating that the shell has become dense and lost its porosity.

Mechanical Characterization of Bubbles Using Nanoindentation. In addition to changes in the shell structure and dimension of the bubbles, the calcination and sintering processes described above significantly change the mechanical properties of individual nanoparticle-shelled bubbles. To fully understand the effect of thermal treatment on the mechanical response of nanoparticle-shelled bubbles, we perform ex situ nanoindentation on single bubbles. ${ }^{28,51}$ We use a $10-\mu$ m-radius $60^{\circ}$ spheroconical indenter, as schematically illustrated in Figure 3a. The indenter and a bubble on a flat substrate are aligned through the main axis perpendicular to the substrate, assuring that the bubble is center-loaded without any sliding during the indentation test. The bubbles are loaded at a constant ratio of loading rate to load $(\dot{P} / P=$ constant $)$ of 0.04 $\mathrm{s}^{-1}$ until failure is detected. Failure is assigned to the first large pop-in event observed on the load-displacement curve (see as example Figure S3 of the Supporting Information). The average diameter and shell thickness of as-assembled, calcined, and sintered bubbles used for all subsequent mechanical characterization are summarized in Table 1. Load-displacement curves are recorded for all experiments and plotted as shown in Figure 3. 
Table 1. Diameter and Shell Thickness of Mechanically Characterized Shelled Bubbles

\begin{tabular}{lcc} 
& diameter, $d(\mu \mathrm{m})$ & shell thickness, $t(\mu \mathrm{m})$ \\
as-assembled bubbles & $40.6 \pm 1.1$ & $2.9 \pm 0.5$ \\
calcined bubbles & $37.8 \pm 1.6$ & $2.7 \pm 0.6$ \\
sintered bubbles & $34.6 \pm 1.1$ & $2.6 \pm 0.6$ \\
\hline
\end{tabular}

Seven individual as-assembled bubbles are tested, and the load-displacement curves are shown in Figure $3 \mathrm{~b}$. The response of these as-assembled bubbles shows nonlinear mechanical behavior (Figure $3 \mathrm{~b}$ ), reaching an average failure load $P_{\mathrm{f}}=3.6 \pm 1.1 \mathrm{mN}$ and an average failure deflection $\delta_{\mathrm{f}}=3.5$ $\pm 0.8 \mu \mathrm{m}$, amounting to an $8.7 \%$ deflection relative to the initial diameter $\left(\delta_{\mathrm{f}} / d\right)$. The energy to failure extracted by integrating the load-displacement curves up to the point of failure, has an average value $U_{\mathrm{f}}=7.7 \pm 3.2 \mathrm{mN} \cdot \mu \mathrm{m}$. Pauchard and Rica previously studied the deformation mechanisms of an elastic spherical shell with a thick wall $(t / R \sim 1 / 10$, where $t$ and $R$ are the thickness and the radius of the shell, respectively), and observed a sharp transition to nonlinear behavior when buckling occurs. ${ }^{52}$ The nonlinear behavior of as-assembled bubbles cannot be tied to such a buckling phenomenon, because the deflection of the bubbles in our test is relatively small $(\delta / t<2$, where $\delta$ is the deflection imposed on the shell). The nonlinear behavior suggests an inelastic response governing the mechanical properties of as-assembled bubbles, which we investigate in more detail below.

Similar nanoindentation tests are performed on calcined and sintered bubbles. Figure $3 \mathrm{c}$ shows the load-displacement responses of five different calcined bubbles tested. In contrast to what is observed for the as-assembled bubbles, the calcined bubbles respond linearly to the applied load, implying a predominantly elastic behavior. The failure load of the calcined bubbles is larger than as-assembled bubbles, reaching an average load of $P_{\mathrm{f}}=6.5 \pm 2.1 \mathrm{mN}$. The average failure deflection of calcined bubbles, however, is significantly smaller than that of the as-assembled bubbles; the average deflection is $\delta_{\mathrm{f}}=1.1 \pm 0.3 \mu \mathrm{m}$, a mere $2.8 \%$ of the initial diameter. The average energy to failure of the calcined bubbles is $U_{\mathrm{f}}=3.6 \pm$ $1.9 \mathrm{mN} \cdot \mu \mathrm{m}$. These results suggest that the calcination process has strengthened the bubble shells, but the absence of organics in the shell causes a more brittle response, reduced deformability, and energy to failure.

Sintered bubbles also respond linearly to nanoindentation, as shown by the nine bubbles tested in Figure $3 \mathrm{~d}$. The sintered bubbles present a significantly higher strength and stiffness compared to calcined and as-assembled bubbles. The average failure load for these sintered bubbles is $P_{\mathrm{f}}=50.3 \pm 16.1 \mathrm{mN}$, an order of magnitude larger than that of calcined ones. It is interesting to note that the deformability of sintered bubbles is also greater than that of calcined bubbles, reaching an average deflection of $\delta_{\mathrm{f}}=2.5 \pm 0.8 \mu \mathrm{m}$, amounting to a $7.2 \%$ of the initial diameter of the bubble. The average energy to failure of the sintered bubbles is $U_{\mathrm{f}}=66.7 \pm 39.4 \mathrm{mN} \cdot \mu \mathrm{m}$. Remarkably, the maximum deflection of the sintered bubbles is quite similar to that of the original as-assembled bubbles, but the energy to failure is an order of magnitude higher. The sintering process has created a shell that is dense and nonporous, which significantly affects the mechanical response of the bubbles to indentation, resulting in high strength, stiffness, large deformability, and exceptionally high energy to failure (tough- ness). The mechanical properties of the bubbles will be summarized and compared later.

Finite Element Analysis of Bubble Nanoindentation Prior to Fracture. The mechanical response of the nanoparticle-shelled bubbles depends significantly on thermal treatment, as illustrated by the nanoindentation experiments. Notably, the sintered bubbles are stiffer, i.e., support higher loads at the same depth of indentation, (and stronger) than the calcined bubbles, which are stiffer than the as-assembled bubbles, as seen in Figure 3. Comparing the load-deflection curves, we see that the as-assembled bubbles display an inelastic behavior. We also observe some variation in the mechanical response of bubbles with the same thermal treatment, as seen in each panel of Figure 3.

To quantify how the mechanical properties of the nanoparticle-shelled bubbles depend on thermal treatments and to explore what accounts for variations in the mechanical response of bubbles with the same nominal size and thermal treatment, we perform finite element analyses (FEA) of the bubbles under nanoindentation. ${ }^{53}$ The FEA requires the inputs of the mechanical properties (elastic moduli and yield strength) of the bubbles. Those properties are estimated by nanoindentation testing on $\mathrm{SiO}_{2}$ nanoparticle films prepared on planar silicon or quartz substrates subjected to the same thermal treatments as the bubbles. Details on the formation of nanoparticle films and their characterization are provided in the Methods section and the Supporting Information. The nanoindentation results of as-prepared nanoparticle films suggest that the as-assembled bubble shell behaves approximately as an elastic-perfectly plastic material, i.e., no hardening beyond yielding. The isotropic von Mises yield criterion is assumed in FEA modeling, in which case the plastic strains are incompressible. As indicated by the nanoindentation results, calcined and sintered films are modeled as ideally elastic. The outer diameter, shell modulus, and thickness of bubbles are adjusted to reflect the experimental values and are given in Table 2. Also, as will be discussed later in more detail,

Table 2. FEA Parameters of Simulated as-Assembled, Calcined, and Sintered Bubbles

\begin{tabular}{|c|c|c|c|c|}
\hline & $\begin{array}{c}\text { shell }^{a} \\
\text { modulus, }^{a} \\
E(\mathrm{GPa})\end{array}$ & $\begin{array}{c}\text { shell } \\
\text { geometry }\end{array}$ & $\begin{array}{c}\text { outer } \\
\text { diameter, } \\
d(\mu \mathrm{m})\end{array}$ & $\begin{array}{c}\text { shell } \\
\text { thickness, } \\
t(\mu \mathrm{m})\end{array}$ \\
\hline \multirow{2}{*}{$\begin{array}{l}\text { as-assembled } \\
\text { bubble }\end{array}$} & 6.9 & uniform & 40.6 & 2.9 \\
\hline & & nonuniform & 40.6 & $\begin{array}{l}2.4 \text { (thin), } \\
3.4 \text { (thick) }\end{array}$ \\
\hline \multirow{2}{*}{$\begin{array}{c}\text { calcined } \\
\text { bubble }\end{array}$} & 13.7 & uniform & 37.8 & 2.7 \\
\hline & & nonuniform & 37.8 & $\begin{array}{l}2.1 \text { (thin), } \\
3.3 \text { (thick) }\end{array}$ \\
\hline \multirow{2}{*}{$\begin{array}{l}\text { sintered } \\
\text { bubble }\end{array}$} & 76 & uniform & 34.6 & 2.6 \\
\hline & & nonuniform & 34.6 & $\begin{array}{l}2 \text { (thin), } \\
3.2 \text { (thick) }\end{array}$ \\
\hline
\end{tabular}

${ }^{a_{T}}$ The elastic moduli are measured on nanoparticle films on planar substrates, as explained in the text.

to study the effect of shell-thickness nonuniformity of each individual bubble, both uniform and nonuniform shells are simulated (see Table 2). The finite element results presented below account for large axisymmetric deflections of the shell under nanoindentation loading. 
a)

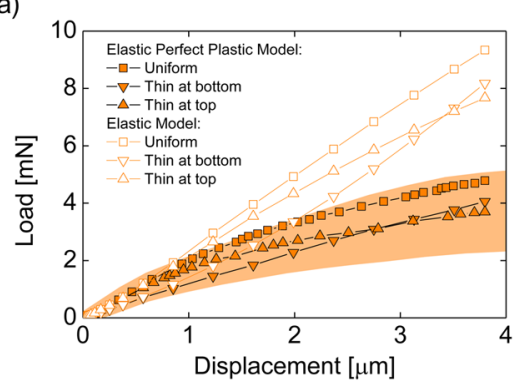

c)

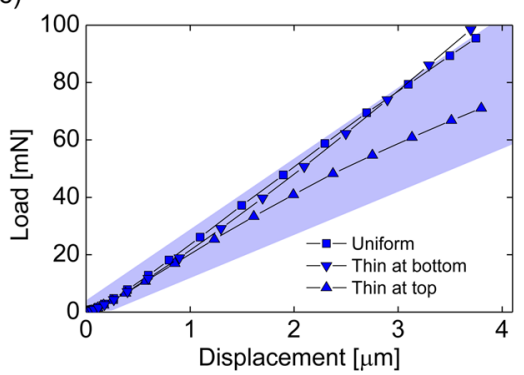

b)

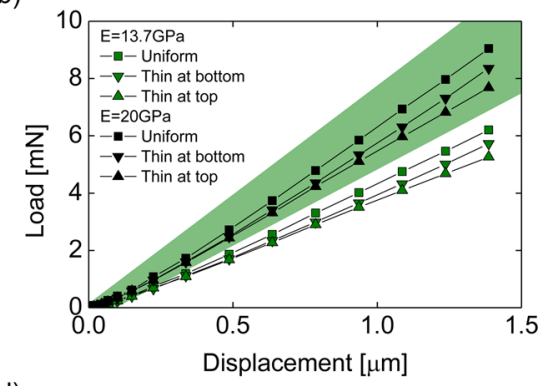

d)

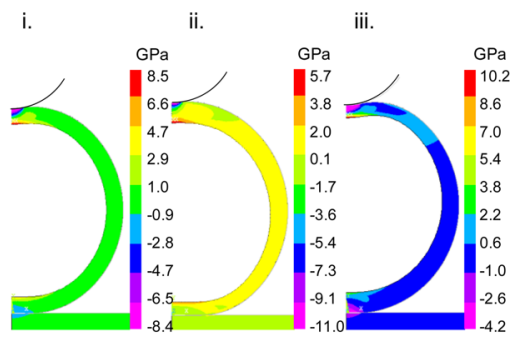

Figure 4. Load-displacement curves simulated using FEA for (a) as-assembled, (b) calcined, and (c) sintered bubbles, respectively, with comparisons to the experimental results (shaded regions). (d) Contour plots from FEA showing first principal stress of a sintered bubble under a spherical indenter at an indentation depth, in each case, corresponding to the average of the first fracture observed in the experimental nanoindentation ex situ tests. Panels i, ii, and iii represent different shell geometries (uniform, thin at bottom, and thin at top, respectively), indicating the differences in the first principal stress distribution along the shell at the maximum indentation depth predicted from the FEA.

The experimental and the FEA-simulated load-displacement curves are shown in Figure $4 a-c$ for each bubble type. For the as-assembled bubbles, two sets of FE simulations were conducted on the basis of pure elasticity and elastic-perfect plasticity, respectively. Figure $4 \mathrm{a}$ shows that compared to the experimental results (shaded region), the elastic FEA displays linear load-displacement behavior and overestimates the load and stiffness of the bubbles for indentations greater than about $1 \mu \mathrm{m}$. However, the elastic-perfectly plastic model with a uniaxial yield stress of $\sigma_{\mathrm{Y}}=118 \mathrm{MPa}$ gives a less stiff response and matches the nanoindentation experiments. These results, in contrast to the nanoindentation of the calcined and sintered bubbles (see Figure 4b,c), indicate that the as-assembled bubbles likely undergo plastic deformation during indentation, accounting for the observed nonlinear deformation. The apparent plastic deformation, we believe, is due to the rearrangement of randomly packed silica nanoparticles within the as-assembled bubble shell under load. Such rearrangement occurs through frictional sliding and causes irreversible deformation, which results in the plasticity of the as-assembled bubbles. 54

The FEA results of indentation on elastic sintered bubbles with a Young's modulus of $E=76 \mathrm{GPa}$ (Figure 4c) display a linear load-displacement response with excellent agreement with the experimental results (shaded area in Figure 4c). The simulation results for the elastic calcined bubbles (Figure $4 \mathrm{~b}$ ) with a Young's modulus of $E=13.7 \mathrm{GPa}$ also predict the linearity of the response; however, these load-displacement curves lie below the experimental curves. By using a slightly higher modulus of $E=20 \mathrm{GPa}$ for the bubble shell, the FEA prediction is in better agreement with the experimental results, indicating that the Young's modulus obtained by characterizing a calcined nanoparticle film on a planar substrate underestimates the stiffness of the calcined bubble shell. Such a discrepancy may be attributed to the difference in the boundary conditions/confinements; nanoparticle films are bound to a substrate, whereas bubble shells are free-standing films of nanoparticle packings. ${ }^{55}$

We hypothesize that variations observed in the loaddisplacement curves for each bubble type, as seen in Figure 3 , can be attributed to the nonuniformity in the thickness of the shell for each bubble and cannot be explained solely by small variations of the bubble diameter or the material properties of the shell. ${ }^{56}$ In fact, recent reports have shown that nonuniformity in the shell thickness of vesicles can drastically change their deformation behaviors under hydrostatic pressure. $^{57,58}$ Also, Carlisle et al. investigated the failure mechanisms of carbon "microballoons" (linear elastic brittle materials) with finite element modeling, predicting a change in failure mode depending on the nonuniformity of the shell. ${ }^{53}$ SEM observation of broken bubbles shows that the bubble shell thickness does vary for each bubble (see, for example, Figure 6, right column), most likely due to the buoyancy of the gaseous core during solvent evaporation from the middle phase (oil) of the $\mathrm{G} / \mathrm{O} / \mathrm{W}$ compound bubbles. The effect of shell thickness variation is approximated by offsetting the two centers of two spherical surfaces that define the shells, incorporating a thin section at the top or at the bottom of the bubble. The thinnest and thickest sections of the bubble shell reflect the standard deviation of the shell thickness summarized in Table 2 .

Load-displacement curves for the nonuniform shells are also extracted from the FEA simulations and compared with the experimental results. Figures $4 a-c$ show the simulated loaddisplacement curves for bubbles with uniform and nonuniform shells for each bubble type. It is interesting to note that, for all bubble types, the stiffness for the nonuniform geometries is predicted to be less than the stiffness of a uniform bubble with a wall thickness equal to the average thickness of the nonuniform ones. Bubbles that are thinnest under the indenter display the least stiff response. That is, at a given depth of indentation, the load required for nonuniform shells is always smaller than that of uniform shells with comparable wall thickness. Shell 


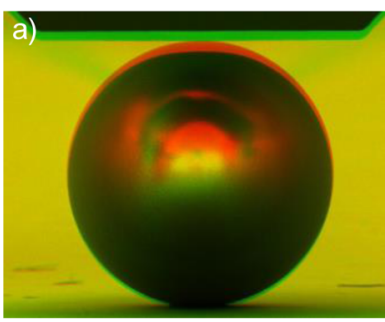

Red channel: before compression Green channel: after compression
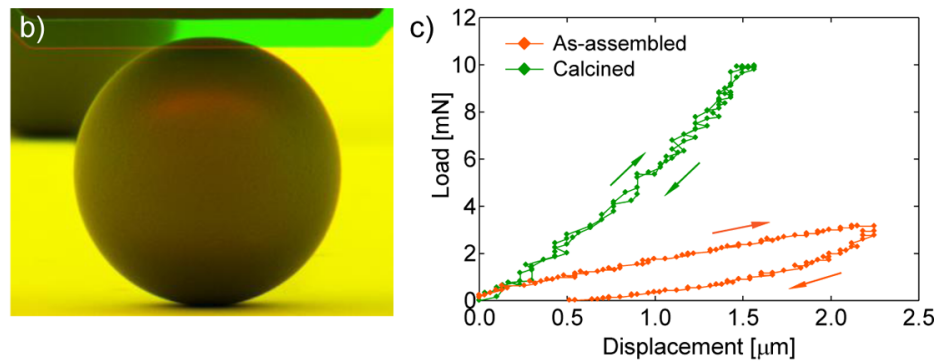

Figure 5. Superimposed SEM images of (a) as-assembled and (b) calcined bubbles before and after load-unload test. (c) Load-displacement curves of the two bubbles after load-unload cycles. Displacement for each bubble is kept low to ensure that no fracture is observed.
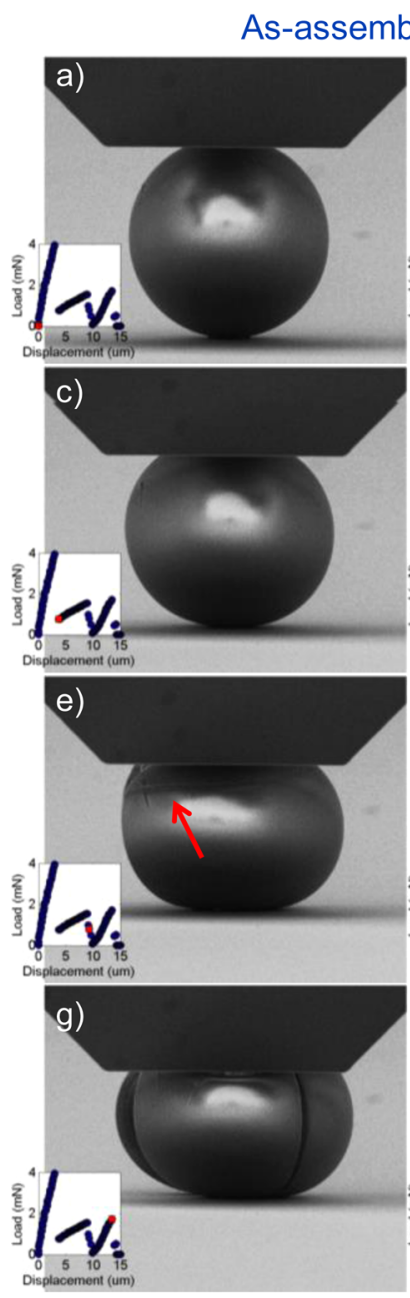
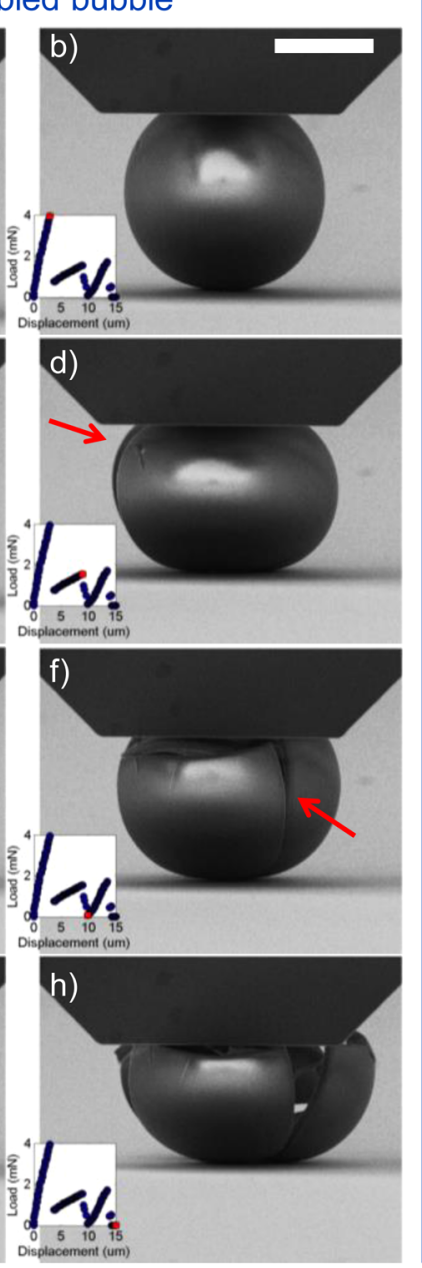
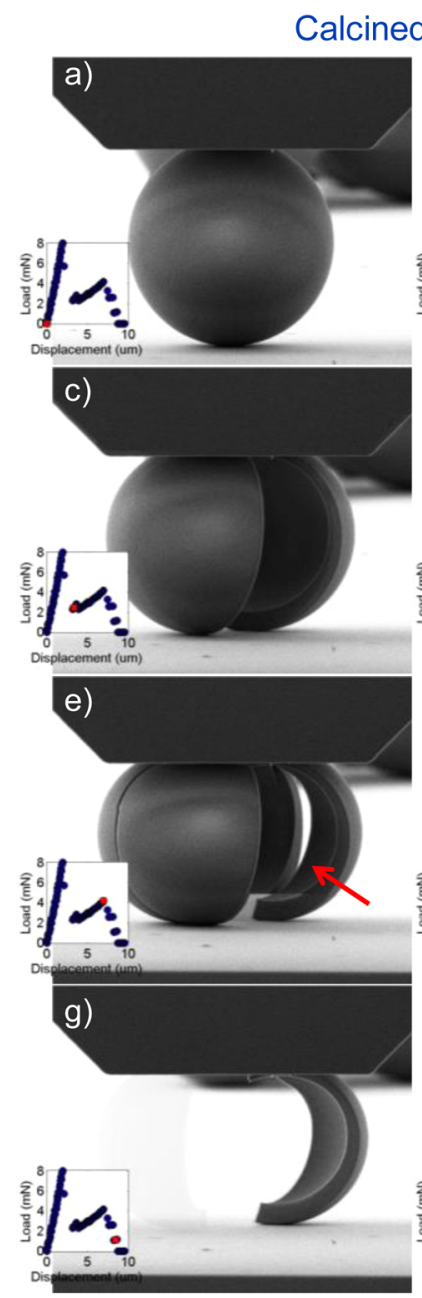
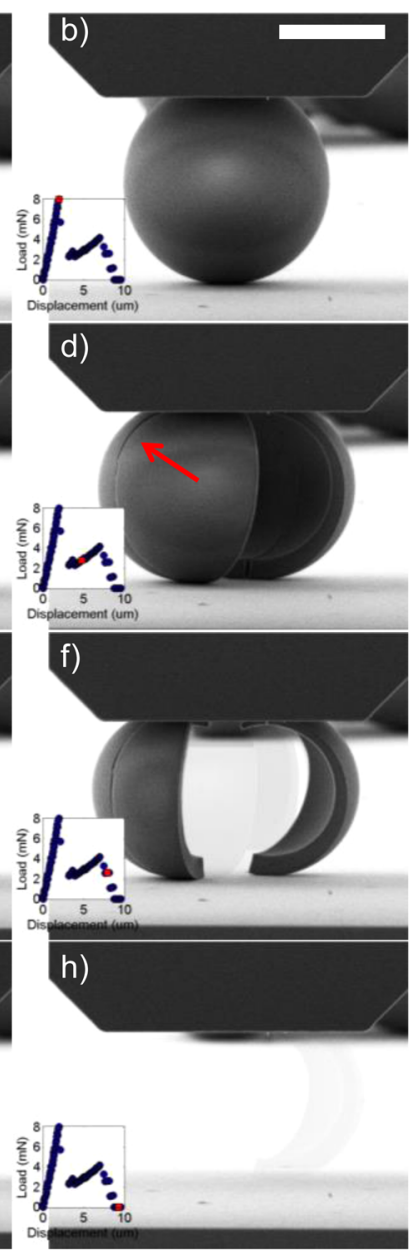

Figure 6. Fracture mechanism of a characteristic as-assembled bubble (left) and calcined bubble (right) from quantitative in situ compression testing with a flat punch; $(\mathrm{a}-\mathrm{h})$ different frames along the experiment. Scale bars $20 \mu \mathrm{m}$. Inset graphs indicate the load-displacement at the specific time. Red arrows point at originated cracks.

thickness nonuniformity also results in greater stress concentrations (see Figure $4 \mathrm{~d}$ and the discussion below), which likely causes fracture to occur in the nonuniform bubbles at smaller indentation depths compared to failure of the uniform bubbles.

The distributions of stresses and strains observed in the FE simulations can provide insight into failure mechanisms. For example, for the sintered bubble, principal stress distributions are plotted in Figure $4 \mathrm{~d}$ at indentation depths that for each case correspond to the average of the first observable fractures. In each case, the maximum principal stress occurs at the inner surface of the shell beneath the initial point of contact with the spherical indenter, regardless of the shell geometry (uniform vs nonuniform thickness). At that location, the maximum principal stress corresponds to a circumferential stress component with respect to the axis of symmetry. That stress would cause a (brittle) crack to initiate along a meridian. The magnitude of the principal stress as well as the stress distribution at the average displacement that causes fracture for each bubble, however, varies noticeably depending on the shell geometry. The magnitude of the maximum principal tensile stresses varies from $\sigma_{\mathrm{I}}=8.5$ to 5.7 and $10.2 \mathrm{GPa}$, for the shell with uniform thickness, the shell that is thin at the bottom, 
a)
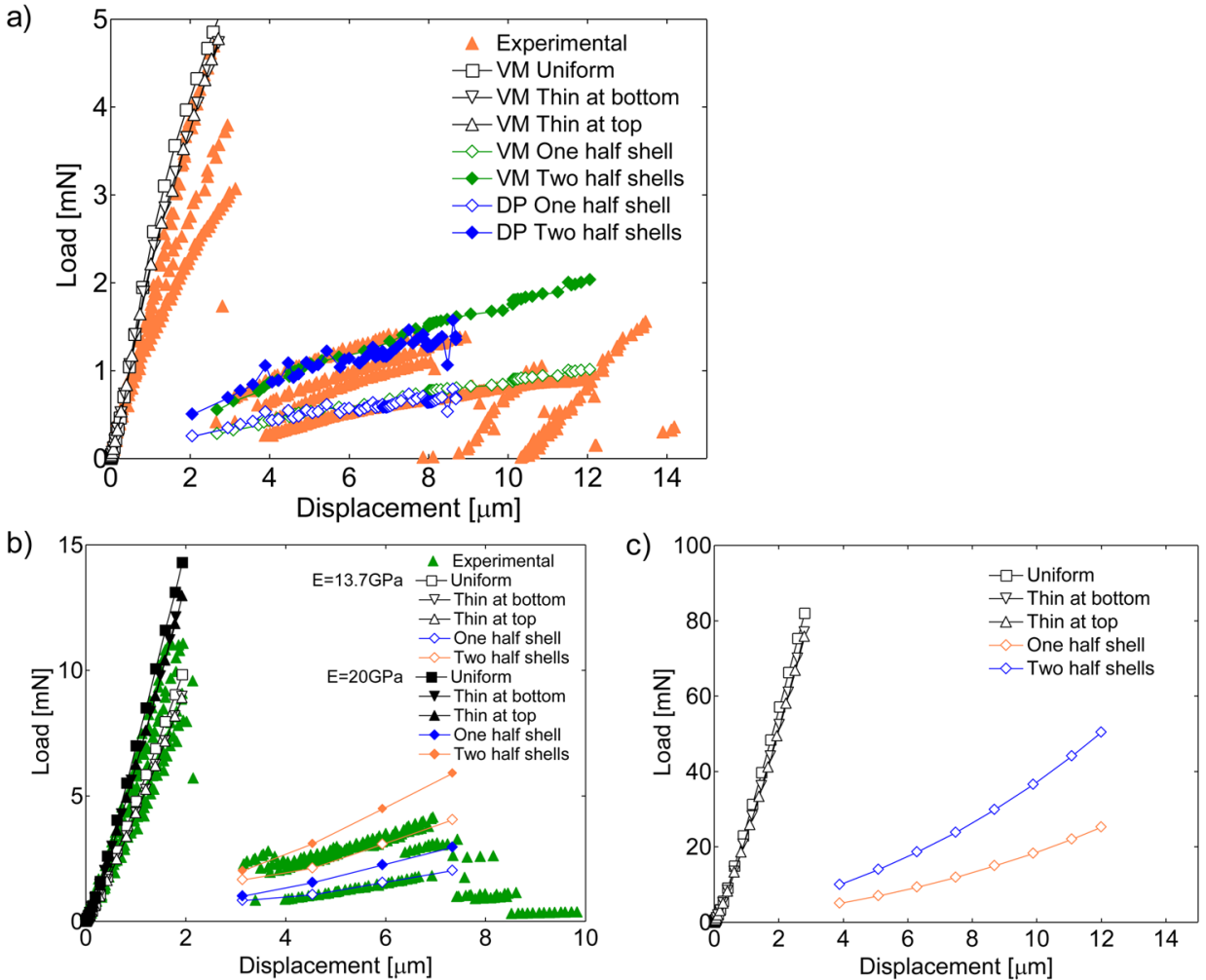

Figure 7. Load-displacement curves of in situ compression of (a) four as-assembled bubbles and (b) four calcined bubbles, including both the first failure event as well as the secondary cracking events that occur on loaded remnants of the fracture shells. FEA results for the first failure event are included in the graphs for three different shell geometries (uniform shell, thin at top, and thin at bottom). FEA results for secondary cracks for uniform shells are also plotted, including the responses when one or two halves of the shell remain under load between the indenter and the substrate. (c) Simulation results for the failure prediction of a sintered bubble lacking experimental values. As-assembled bubbles (a) are simulated with elastic-perfectly plastic von Mises (VM) and Drucker-Prager (DP) models. Calcined (b) and sintered (c) bubbles are simulated with an elastic model.

and the shell that is thin at the top, respectively (Figure $4 \mathrm{~d}$ ). These differences in the magnitude of the maximum principal stresses suggest that fracture would occur at different loads for different geometries. For the different bubble types, asassembled, calcined, and sintered, the magnitude of the maximum principal stress at the average failure deflection for uniform shells are $\sigma_{\mathrm{I}}=135,528 \mathrm{MPa}$ (for $E=13.7 \mathrm{GPa} ; 769$ $\mathrm{MPa}$ for $E=20 \mathrm{GPa}$ ), and $8.5 \mathrm{GPa}$, respectively, which is an indicative of the critical fracture strength of the shell materials.

Quantitative in Situ Mechanical Characterization of as-Assembled and Calcined Bubbles. Quantitative in situ mechanical characterization methods provide invaluable information that cannot be readily obtained via ex situ techniques (e.g., nanoindentation) by allowing for simultaneous real-time monitoring of deformation and failure processes and the acquisition of load-displacement information during deformation. ${ }^{59,60}$ Here we directly observe the mechanical response and failure of individual as-assembled and calcined bubbles under displacement-controlled compression. A flat indenter installed inside a scanning electron microscope (SEM) chamber is used to apply uniaxial compression at a constant nominal strain rate $\dot{\delta} / d=0.001 \mathrm{~s}^{-1}$ on individual bubbles on a planar substrate $(\mathrm{Si}$ wafer). The differences between the ex situ and the in situ methods used in this study are summarized in the Supporting Information.

We directly observe the plasticity of an as-assembled bubble by applying and removing a compressive load prior to fracture. As can be seen from the overlaid SEM images of the as- assembled bubble after multiple load-unload tests (Figure 5a), the bubble undergoes a permanent deformation. In contrast, a calcined bubble returns to its original shape without any observable permanent deformation (Figure $5 b$ ), indicating a purely elastic response. The quantitative load-displacement curves for the two bubbles also show that the as-assembled bubble permanently deforms, whereas the calcined bubble completely recovers its original shape after one load-unload cycle (Figure 5c). Also noteworthy is the fact that the asassembled bubbles display hysteresis in the load-displacement curves and permanent deformation upon unloading, as seen in Figure 5c.

We also compare the failure mechanisms of as-assembled and calcined bubbles by subjecting them to large compressive loads. Figure 6 presents a collection of movie frames showing the compression of an as-assembled bubble (left) and a calcined bubble (right) between two planar surfaces (full movies are available in the Supporting Information). Images on the left column of Figure 6 show that an as-assembled bubble undergoes a significant deformation before the indenter moves downward suddenly; this sudden downward movement coincides with a precipitous drop in the load-displacement curve (from $b$ to $c$ in the left column of Figure 6), indicating fracture of the as-assembled bubble. The crack, although not clearly visible because it runs parallel to the viewing plane, vertically splits the as-assembled bubble into two halves (indicated by the red arrow in Figure 6d of the left column). Following this first crack, the fractured bubble remains in 
contact between the two plates. We believe that this first crack initiates at the pole adjacent to the flat indenter. After the first fracture of as-assembled bubbles, the two split halves continue to deform significantly under further compression (additional 5 $\mu \mathrm{m})$ before a second fracture initiates. Interestingly, the second crack consistently propagates horizontally (parallel to the two plates), denoted by the red arrow in Figure 6e left, in all of the six as-assembled samples we test. Additional vertical cracks appear as the fractured bubble shell is further compressed, indicated by the red arrow in Figure $6 \mathrm{f} \mathrm{left.}$

The failure of stiffer calcined bubbles is quite different from that of as-assembled bubbles. After a calcined bubble comes in contact with the flat indenter, it undergoes smaller deformation than the as-assembled bubble before the first failure (from $b$ to c in the right column of Figure 6) is observed, which also corresponds to a vertical crack. With continued loading, a second crack initiates and propagates in a vertical direction (perpendicular to the substrate) as indicated by the red arrow in Figure 6d right, which is strikingly different from the secondary horizontal cracks observed in the as-assembled bubbles. Both the first and second cracks in the calcined bubble initiate at a smaller deformation than the corresponding cracks in the as-assembled bubbles. Furthermore, subsequent cracks in the calcined bubble are all formed in the same way (red arrow in Figure 6e right) running vertically through the shell. The fractured pieces of the shell are expelled out of the field-of-view due to the large elastic energy accumulated in the broken shell, which is not the case for the plastically deforming as-assembled bubbles.

The first failure event for both the as-assembled and the calcined bubbles occurs through the propagation of a vertical crack between the points of contact of the bubble shell with the plates. As noted, this result is consistent with the FEA results shown in Figure 4d, in which the maximum first principal stress is always observed beneath the indenter, and also with previous reports that studied the failure mechanism of elastic microballons. ${ }^{53,61}$ However, the secondary cracking that develops after the first (vertical) fractures is strikingly different for the two types of bubbles. Understanding the modes of subsequent failure (i.e., vertical vs horizontal secondary cracks) after the first cracks in these bubbles could be of importance in applications involving composites, because once bubbles fail with horizontal cracks, their ability to bear compressive loads will be significantly compromised. ${ }^{62}$ The load that the asassembled bubble can bear after the formation of the second crack indeed decays to $\sim 0$, as seen in Figure $6 f$, left. In contrast, the fractured pieces of the calcined bubbles are able to withstand substantial load after the formation of multiple vertical cracks (Figure 6e-g, right). Nevertheless, the fracture mechanisms of free-standing bubbles under compression may be different from the mechanisms of bubble failure in a composite material.

The quantitative load-displacement responses of the two types of bubbles obtained using in situ compression tests are consistent with the results from ex situ nanoindentation using the spherical indenter. As-assembled bubbles respond nonlinearly (Figure 7a), whereas the response of calcined bubbles is nearly linear (Figure $7 \mathrm{~b}$ ). The breaks in the curves with significant load drops correspond to successive cracking events. The average failure loads, at the onset of the first cracking event of as-assembled and calcined bubbles, are $P_{\mathrm{f}}=3.9 \pm 0.7$ and 9.9 $\pm 1.4 \mathrm{mN}$, respectively. The average failure deflections are $\delta_{\mathrm{f}}=$ $2.7 \pm 0.4$ and $2.0 \pm 0.2 \mu \mathrm{m}$, amounting to an $6.7 \%$ and $5.3 \%$ relative deflection $\left(\delta_{\mathrm{f}} / d\right)$, respectively, indicating a larger deformability by the as-assembled bubbles compared to the calcined bubbles, as one would expect. The average energies to failure of as-assembled and calcined bubbles are $U_{\mathrm{f}}=5.5 \pm 0.9$ and $9.6 \pm 1.5 \mathrm{mN} \cdot \mu \mathrm{m}$, respectively. The difference in the shape of the indenters in the two tests, spheroconical for nanoindentation and flat for in situ tests, may have resulted in the different values of energies to failure obtained from in situ and ex situ measurements. The results from in situ testing will be summarized and compared with ex situ results later.

Finite Element Analyses of in Situ Experiments. Using FEA, we predict the overall load-displacement curves of both the as-assembled and calcined bubbles, as shown in Figure 7a,b. The load-displacement behavior is predicted for the full bubble under flat plate indentation (axisymmetric) showing consistency with the experimental results. As in the previous FEA, the as-assembled bubbles are modeled as an elasticperfectly plastic von Mises material. The calcined bubbles are modeled as ideally elastic, once again using two different Young's moduli of $E=13.7$ and $20 \mathrm{GPa}$ (Figure 7b). Both simulation results agree well within the range of experimental tests. Although we cannot perform in situ testing on sintered bubbles because they are too stiff and strong for our in situ indentation system, we are able to predict their response using FEA with a Young's modulus of $E=76 \mathrm{GPa}$, as shown in Figure $7 \mathrm{c}$, demonstrating the utility of our computational approach to understand the mechanical response of these small hollow structures.

To better understand the nature of secondary cracking and the load-displacement response of fractured bubbles under compression, we carry out a series of FE calculations of the halfshell that arise after the first fracture. The load-displacement predictions for the secondary cracking depend on whether the two half-shells remain in full contact with the indenter or only one of the two half-shells are under load after the first fracture event (i.e., when the fractured pieces of the shell are expelled out of the field-of-view due to the large elastic energy accumulated in the broken shell or when a piece loses contact between the flats during the further compression). In the case of the calcined half-shell, the computed first principal stress distribution is a maximum under the indenter (see Figure S6 of the Supporting Information), and that corresponds to a circumferential normal stress component that is consistent with the vertical secondary cracks in the in situ tests. Consequently, we believe that the mechanism of cracking in the calcined bubbles is reasonably well understood.

The mechanism that leads to the formation of horizontal cracks in the half-shells of as-assembled bubbles is a more challenging problem. The role of plasticity in redistributing the stress in as-assembled bubbles during compression may provide insight. We carry out a series of FE simulations on a hemispherical as-assembled shell. The first set of calculations is based on the von Mises elastic-perfectly plastic material model. Although those results can reasonably predict the overall load-displacement curves (see Figure 7a), they do not display definitive trends with respect to distributions of stress and strain components throughout the bubble that can explain the horizontal cracking. At levels of indentation that are consistent with the initiation of the horizontal crack, results from the FE calculations for the von Mises material model are studied in detail. For example, we compute and plot the components of strain in spherical coordinates (not presented for brevity) to investigate if a concentration in the component 
a)

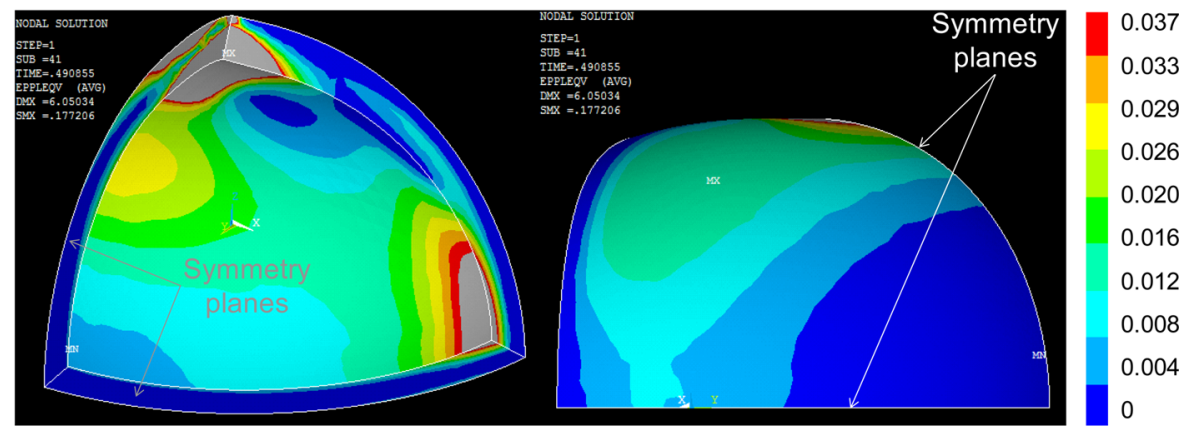

b)

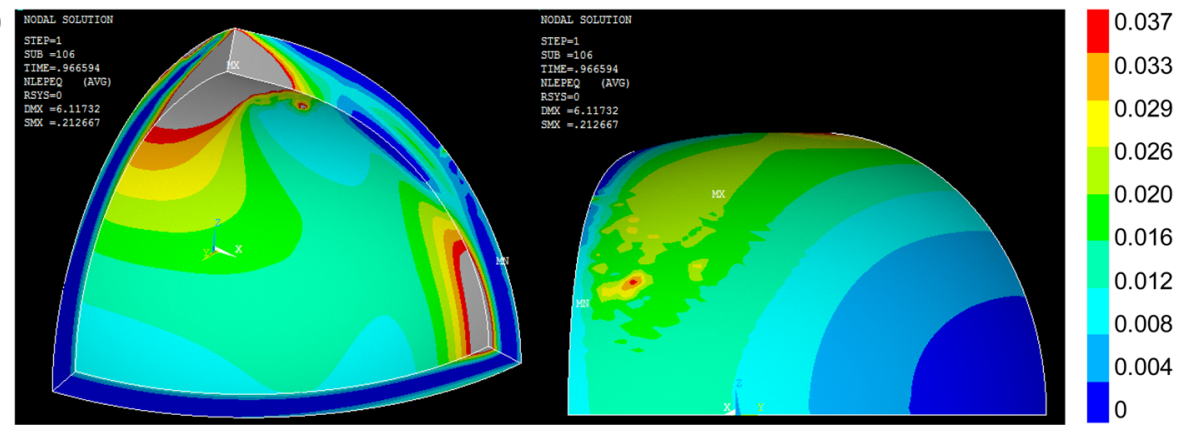

Figure 8. Finite element simulation of an as-assembled half-bubble modeled as a von Mises material (a) and a Drucker-Prager material (b). In each case, the appropriate effective plastic strain is plotted over the inner and outer surfaces. Note the elevation of plastic strain near the midmeridian plane for the Drucker-Prager material.

a)

c)
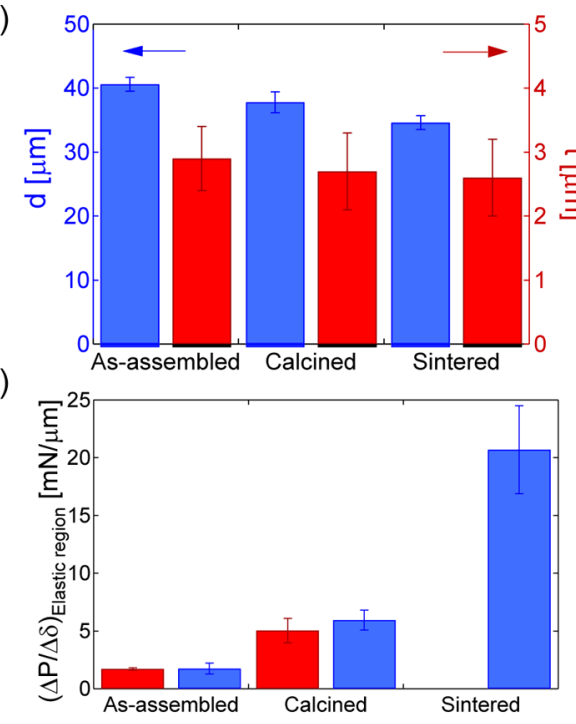

e)

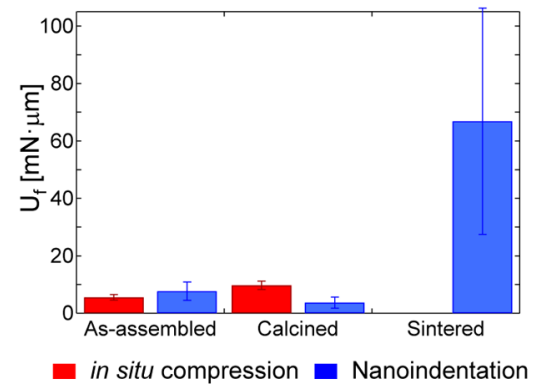

b)

d)
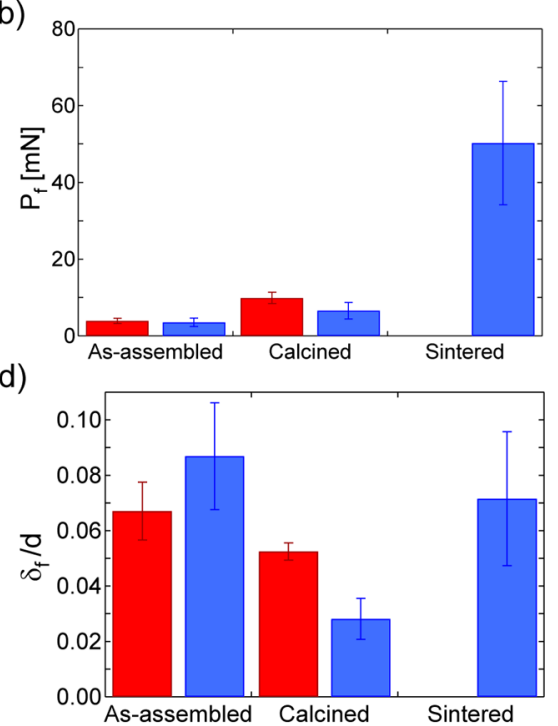

Figure 9. (a) In blue, diameter, $d$, and in red, shell thickness, $t$, of as-assembled, calcined and sintered bubbles. (b) Average failure load of bubbles at fracture, $P_{\mathrm{f}}$, as measured by the maximum load registered before the first crack. (c) Average stiffness of bubbles, $\Delta P / \Delta \delta$, computed from the slope of the load-displacement curves in the elastic regions. For as-assembled bubbles the elastic region is considered at small deflections of $\delta<500 \mathrm{~nm}$. (d) Average maximum deflection of bubbles, $\delta_{\mathrm{f}} / d$, measured just before fracture, normalized by the initial diameter of the bubble. (e) Average energy to failure, $U_{\mathfrak{f}}$ computed from the area under the load-displacement curves before the first fracture. Red and blue bars in parts b-e correspond to in situ compression results and nanoindentation results, respectively. 
a)

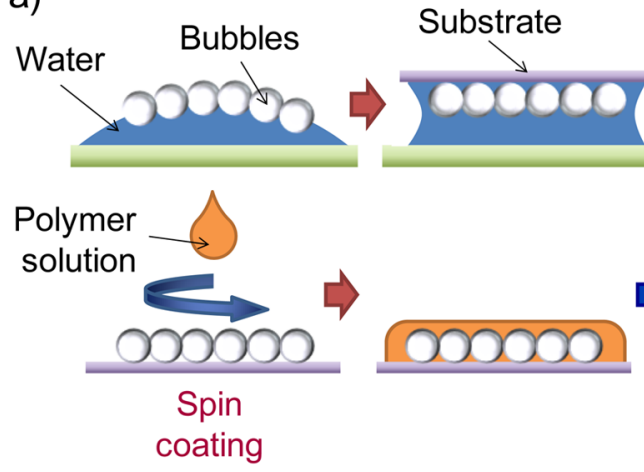

c)

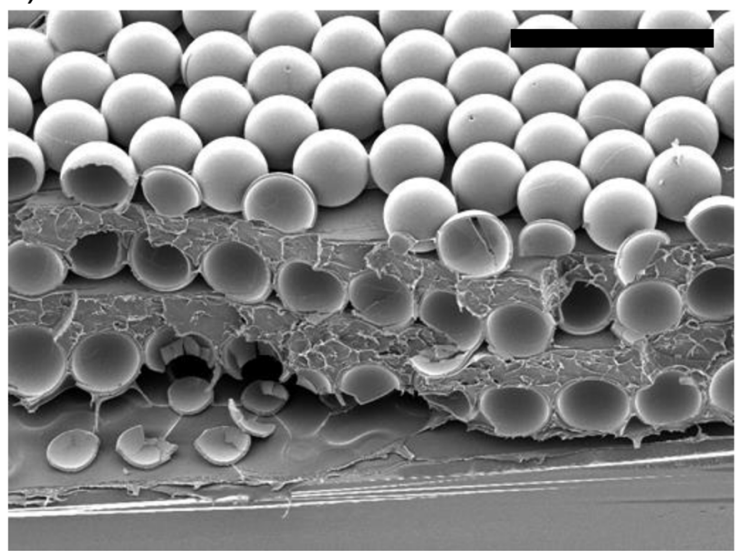

b)

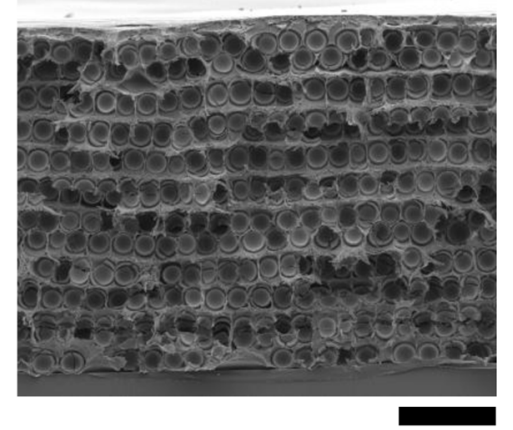

d)

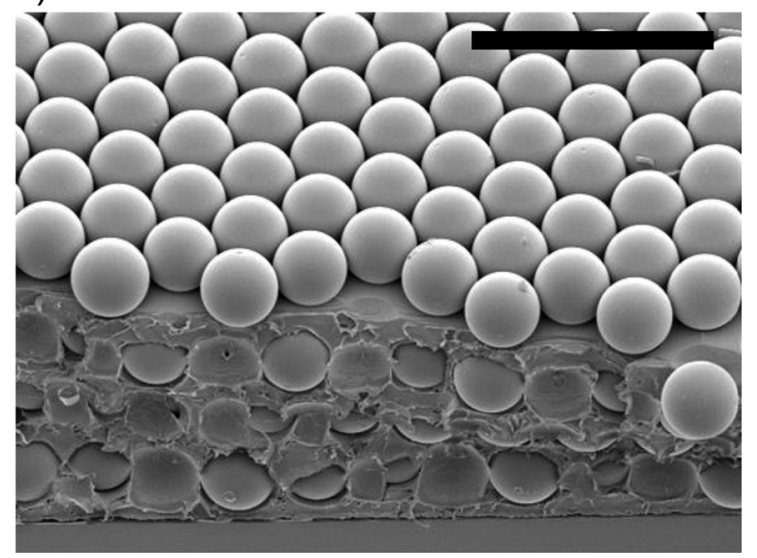

Figure 10. (a) Layer-by-layer (LbL) method used for the generation of polymer-bubble composites. (b) LbL structure composed of 10 bilayers for a total thickness of approximately $1 \mathrm{~mm}$. (c) LbL structure made of as-assembled bubbles and polystyrene. (d) LbL structure made of sintered bubbles and polystyrene. Scale bars $=200 \mu \mathrm{m}$.

of strain acting perpendicular to the incipient horizontal fracture surface exists. We also monitor stress components including the von Mises effective stress (Figure S7a of the Supporting Information) and the hydrostatic pressure (Figure S8a of the Supporting Information) as well as the effective plastic strain (Figure 8a). None of those measures can explain the initiation of a horizontal crack in the as-assembled bubble on a surface that is roughly one-half a bubble radius below the indenter.

A few points about plasticity models are warranted at this point. The von Mises yield criterion and its associated flow rule are independent of hydrostatic pressure, which is a good approximation for a fully dense, typically crystalline material. On the other hand, the as-assembled nanoparticle-shelled bubbles likely display significant pressure sensitivity in yielding and plastic flow due to sliding between the nanoparticles. Another classical model, originally developed for granular materials and soils, is the Drucker-Prager yield criterion, ${ }^{63}$ which adds a pressure-dependent term to the von Mises effective stress. Furthermore, the associated plastic flow rule for the von Mises model is incompressible, whereas the DruckerPrager model includes plastic dilation. Finite element simulations using the latter model are considered below (see the Appendix for a detailed description of the Drucker-Prager model).

The overall load-displacement curves calculated using the Drucker-Prager model are similar to those predicted using the von Mises model (see Figure 7a, DP vs VM curves), and both are in good agreement with experiments. Nevertheless, the predicted distributions of stresses and strains display considerable differences, as one might expect due to the difference in incompressible and dilatant plastic flow. We investigate and compare various components of stresses and strains and find some significant differences. Comparisons of the effective plastic strains for each model are plotted in Figure 8 for asassembled half-bubbles compressed up to a deflection of $9 \mu \mathrm{m}$, which approximately corresponds to the initiation of the horizontal crack, averaged for the four specimens from the in situ tests. Given that the deformations on the outer surface of the shell are predominately tensile, while they are predominately compressive on the inner surface, we expect the secondary cracking to initiate on the outer surface. As seen in Figure 8a for the von Mises material, the magnitude of the effective plastic strain on the outer surface is less than 0.016 , except right under the indenter, while the effective strain for the Drucker-Prager material (Figure $8 \mathrm{~b}$ ) reaches a significantly higher level, up to 0.037 , in a region near the midmeridian plane of the half-shell at a latitude near where horizontal cracks are observed to form. Those differences, which occur at approximately the same overall load levels (see Figure 7a), are significant and indirectly lead to the conclusion that pressuresensitive yielding is characteristic of the as-assembled bubbles, as one would expect. Nevertheless, further detailed studies beyond the scope of these analyses are required, keeping in mind that the Drucker-Prager model is a simple extension of the von Mises model to include pressure sensitivity and plastic dilation. Other mechanistically based, dilational plasticity models have been developed, but their consideration is beyond 
the scope of this work. We also note that as-assembled bubbles likely display rate-dependent mechanical behavior due to the presence of organic materials, which could affect the deformation and failure mechanisms under different loading conditions (i.e., strain rate, stress relaxation). ${ }^{54}$

Summary of Mechanical Characterization. The results of mechanical characterization from the ex situ and in situ indentation tests are summarized for comparison in Figure 9. As seen in Figure 9a, the bubble outer diameter and the shell thickness decrease upon thermal treatment due to the removal of the organics (i.e., PVA and the organic layer on nanoparticle surface) and the decrease in the shell porosity. The failure load significantly increases with the thermal treatment temperature (Figure 9b); that of the calcined bubbles is twice as large as that of the as-assembled ones, and the sintering process strengthens the bubbles by another order of magnitude. A similar trend is observed for the stiffness of the shelled bubbles (Figure 9c). The measure of the stiffness can be extracted from the slope of the elastic regime in each load-displacement curve. While the calcined and sintered bubbles show linear responses, making it straightforward to obtain the slopes, the as-assembled bubbles show nonlinear behavior; therefore, we use linear regression between 0 and $500 \mathrm{~nm}$ deflection $\left(R^{2} \sim 0.997\right.$ for ex situ nanoindentation, and $R^{2} \sim 0.957$ for in situ tests) to estimate their stiffness. Sintered bubbles present the largest stiffness, an order of magnitude larger than the as-assembled bubbles, followed by the calcined bubbles, as shown in Figure 9c.

The energy to failure, i.e., toughness, and the fracture strain, which correlate with the failure deflection (compression) of the bubbles in our experiments, are important properties of the shelled bubbles. Those properties, together with strength and the stiffness, will control the mechanical response and damage tolerance of the composites. ${ }^{30,62,64}$ The average maximum deflection at failure normalized by the initial outer diameter of the bubble is analogous to strain-to-failure and allows us to compare the deformability of the three different bubbles (Figure 9d). As-assembled bubbles undergo a relatively large deflection at failure compared to calcined bubbles. Indeed, the presence of organics in the shell allows the as-assembled bubble to undergo relatively large inelastic (plastic) strains, which increases the bubble ductility. Interestingly, deflection at failure increases when bubbles are treated at $1200{ }^{\circ} \mathrm{C}$, likely due to the large strengthening effect of the fusion of silica nanoparticles and also the elimination of the small defects that porosity introduces in the calcined bubbles. Because of these factors, the energy to failure of the sintered bubbles is significantly greater than those of the as-assembled and calcined bubbles, as seen in Figure 9e. Both the high deformability and high strength of the sintered bubbles impart an exceptional ability to absorb energy before undergoing fracture. A major difference in the behavior of the sintered bubbles and that of the as-assembled bubbles, of course, lies in the fact that the sintered bubbles are purely elastic; thus, bubbles are able to fully recover their original shape, whereas as-assembled bubbles undergo plastic and thus irreversible deformation.

Mechanical Response of Bubbles in Polymer-Bubble Composites. The results from the mechanical testing of individual bubbles presented above clearly show that thermal treatments have significant impact on the mechanical properties of nanoparticle-shelled bubbles. We expect these bubbles to behave very differently when they are incorporated into a polymer matrix. To illustrate the difference in the mechanical responses of the bubbles, we generate bubble-polymer composites using layer-by-layer (LbL) deposition. As shown in Figure 10a, bubbles are deposited on a silicon wafer using a process analogous to the Langmuir-Schaefer technique for nanoparticle printing. ${ }^{65}$ Subsequently, a solution of polystyrene is spin-coated on top of the dried layer of bubbles. Another layer of bubbles is deposited atop the polymer layer. This process is repeated to construct the LbL structure, as shown in Figure $10 \mathrm{~b}$. A qualitative comparison of the response of the bubbles under a large mechanical stress can be made by cleaving the LbL structure atop the Si wafer. While a crack propagates through the bubbles in the composite with asassembled bubbles (Figure 10c), the crack propagates around the bubbles in the sample with sintered bubbles (Figure 10d). This indicates that the weakest link in as-assembled bubblepolystyrene composite is the bubbles themselves, whereas the weakest link in the sintered bubble-polystyrene composite is the bubble-matrix interface. The deformation mechanism of the bubble reinforced polymer composites are currently under investigation.

\section{CONCLUSIONS}

Here, we report the generation of nanoparticle-shelled bubbles and their structure-mechanical property relationship. Nanoparticle-shelled bubbles are generated using microfluidics, and their structure and mechanical properties are tailored by calcination and sintering processes. Both ex situ and in situ mechanical testing show that the mechanical properties and deformation/failure modes of these bubbles depend considerably on the thermal treatment conditions. In fact, the strength of the bubbles is increased by more than an order of magnitude upon sintering. As-assembled bubbles exhibit an inelastic response with significant plasticity afforded by the organic materials in the bubble shell. Interestingly, while the deformability of calcined bubbles is diminished due to the loss of organics, the deformability of sintered bubbles is close to that of as-assembled bubbles, owing to the largely enhanced elastic range. We also find that the shell geometry has a significant impact on the response of these bubbles under load, as shown by finite element analysis. In addition, we use FEA to study the secondary cracking events on as-assembled and calcined bubbles, which experimentally are observed to be horizontal and vertical, respectively. The tendency for horizontal cracking of as-assembled half-bubbles is consistent with the predictions of the elastic-perfectly plastic DruckerPrager material model that accounts for pressure-sensitive yielding and plastic dilation, whereas the use of a perfect elastic Von Mises material model, which is appropriate for the calcined half-bubbles, predicts the observed vertical cracking in that case. In addition, we show that it is possible to generate composites using bubbles with different structure and mechanical properties. The failure mode of the as-assembled and sintered bubbles in a polymer-bubble composite is shown to directly depend on the mechanical properties of individual bubbles. The possibility of changing the mechanical properties of nanoparticle-shelled bubbles makes these hollow particles attractive candidates as fillers for the generation of lightweight materials that need to satisfy different mechanical requirements. In addition to tailoring the mechanical properties of bubbles and the composites containing these bubbles, the incorporation of various functional nanoparticles such as magnetic, semiconducting, and plasmonic nanoparticles into the bubble shell will enable the formation of lightweight materials with specific functionality. ${ }^{66-70}$ 


\section{METHODS}

Generation of Nanoparticle-Shelled Bubbles. Nanoparticleshelled bubbles are generated using a glass microfluidic device that combines coflow and flow-focusing geometry, as previously reported. ${ }^{31}$ The three immiscible fluid phases used are nitrogen (AirGas, Inc.) as the inner phase, hydrophobic silica nanoparticles (15 nm average diameter) suspended in toluene (Nissan Chemical Industries, Ltd.) at an approximate concentration of $28 \mathrm{wt} \%$ as the middle phase, and an aqueous solution containing poly(vinyl alcohol) (PVA, 87-89\% hydrolyzed, average MW $=13000-23000$, Sigma-Aldrich Co. LLC) at a concentration of $2 \mathrm{wt} \%$ as the outer phase. The geometry of the gas-in-oil-in-water $(\mathrm{G} / \mathrm{O} / \mathrm{W})$ compound bubbles is controlled by tuning the flow rates during the microfluidic generation. The shell thickness and diameter of the bubble are tuned to be above a critical value to ensure that the bubbles remain stable upon drying. ${ }^{32}$ The G/ $\mathrm{O} / \mathrm{W}$ compound bubbles are collected in a convex air-water interface, forming a monolayer of bubbles to facilitate the fast evaporation of the toluene in the middle phase. When the nanoparticle-shelled bubbles are formed, they are washed to remove the excess of PVA by exchanging the water in the collecting container three times. A monolayer of as-assembled bubbles is formed by drying a drop of bubble suspension on a piece of silicon wafer.

Thermal Treatment: Calcination and Sintering Processes. Thermal treatment is performed on the monolayer of dried bubbles on a silicon wafer. The bubbles are calcined at $700{ }^{\circ} \mathrm{C}$ using a Thermo Scientific Thermolyne furnace benchtop muffle type F47900 for approximately $3 \mathrm{~h}$. The sample is then cooled down to room temperature by removing the sample from the furnace. For the generation of sintered bubbles, a monolayer of as-assembled bubbles on a silicon wafer is sintered at $1200{ }^{\circ} \mathrm{C}$ for a short period of time. The furnace is allowed to reach $1200{ }^{\circ} \mathrm{C}$ and then is turned off to cool down; when temperature in the furnace cools down to $700-800{ }^{\circ} \mathrm{C}$, the sample is taken out of the furnace.

Characterization of Bubbles and Bubble Shells. As-assembled, calcined, and sintered bubbles are resuspended in water to verify their integrity and geometry. Calcined bubbles easily detach from the silicon wafer by placing a drop of water. Calcined bubbles allow water to permeate through the shells into their cores due to their porosity and change in their wettability (see Supporting Information, Figure S2). Sintered bubbles occasionally remain attached to the substrate after the sintering process. The detachment of the bubbles from the substrate is achieved by introducing the silicon wafer with the attached bubbles in a glass vial containing DI water and performing a brief ultrasonication $(<1 \mathrm{~s})$ using a bath ultrasonicator (9.5 L FisherScientific FS-110D). Approximately, $90 \%$ of the bubbles detach from the Si wafer without significant damage.

Characterization of the as-assembled, calcined, and sintered bubbles is performed by optical microscopy using a Zeiss Axioplan 2 upright microscope equipped with a Q-imaging Retiga 2000R Fast 1394 CCD digital camera. Images of the monolayer of dried bubbles atop a silicon wafer before and after the heat treatment are taken in reflection mode while resuspended bubbles are imaged in transmission mode. ImageJ software is used for the image analysis to determine average bubble diameter, size distribution, stability, and permeability. Scanning electron microscopy (SEM) images are taken using a FEI Quanta 600 FEG ESEM at $5-10 \mathrm{kV}$. Average shell thickness values are determined by averaging measurements obtained from the analysis of SEM images taken on fractured bubbles.

Nanoindentation. Nanoindentation on as-assembled, calcined, and sintered bubbles is performed using a Nano Indenter G200 from Agilent Technologies Inc. A $10 \mu \mathrm{m}$ radius $60^{\circ}$ spheroconical rigid indenter is used to obtain load-displacement curves using a constant ratio of loading rate to load $(\dot{P} / P=$ constant $)$ of $0.04 \mathrm{~s}^{-1}$. Thermal drift correction is performed. For nanoindentation tests, the bubbles are deposited onto Si wafers. The Si wafer plates are much stiffer than the bubbles and undergo negligible deformation.

In Situ Compression. Quantitative in situ mechanical testing of bubble specimens is performed using a novel custom-built micro- and nanomechanical testing system installed in a high-resolution field- emission SEM (FEI Quanta 600F). ${ }^{60}$ The testing platform consists of three primary components: (i) a stiff piezoelectric actuator operated in closed-loop control mode ( $1 \mathrm{~nm}$ resolution), enabling displacementcontrolled testing; (ii) a six degree-of-freedom closed-loop nanopositioning system (SmarAct SmarPod, with $1 \mathrm{~nm}$ and $1 \mu \mathrm{rad}$ resolution); and (iii) a capacitive-based force-sensing probe (Femtotools FT-S10000 microforce sensing probe, with $0.5 \mu \mathrm{N}$ resolution at $10 \mathrm{~Hz}$ acquisition rate). A square $\mathrm{Si}$ flat punch $(50 \times 50$ $\mu \mathrm{m})$ at the tip of the load cell is used for compression testing.

Special considerations are made during testing to eliminate the effects of misalignment between the flat punch (and thus the load cell axis) and the testing specimen. Alignment is achieved by using the nanopositioning stage. In-plane alignment is relatively straightforward and achieved by rotation and translation of the tip relative to the specimen with feedback based on SEM observation. We achieve the optimal out-of-plane alignment by maximizing the contact stiffness as a function of rotation angle during low load indentation experiments on the substrate adjacent to bubble specimens. Compression tests are operated in displacement control to achieve displacement rates of approximately 40 and $37 \mathrm{~nm} / \mathrm{s}$, for as-assembled and calcined bubbles, respectively, and SEM images are simultaneously acquired. For in situ compression tests, the bubbles are deposited onto Si wafers. The Si wafer plates are much stiffer than the bubbles and undergo negligible deformation.

Finite Element Analysis. ANSYS 13.0 commercially available software is used for the simulation of the mechanical characterization of the single bubbles. Due to the spherical geometry of the shelled bubbles and the contact symmetry, axisymmetry is assumed and the simulations are performed in the two-dimensional space using axisymmetric quadratic elements (PLANE183, higher order 2-D, eight- or six-node element). The mesh for a cross section of an axisymmetric bubble (see Figure 4d) comprises more than 5000 elements and 15000 nodes, with at least 16 elements radially spanning from the inner shell surface to the outer surface. The substrate is designed as a $175 \times 175 \mu \mathrm{m}^{2}$ elastic silicon block (Young's modulus, $E$ $=162 \mathrm{GPa}$ and Poisson's ratio, $\nu=0.22$ ) which is large enough to have a negligible boundary effect on the modeling. For the simulations of the ex situ nanoidentation, a $10-\mu \mathrm{m}$-radius spherical rigid tip is used for the simulation of the indenter. For the simulations of the in situ compression, a flat rigid tip is used for the simulation of the indenter. The contacts between the bubble and the indenter and the bubble and the substrate are assumed frictionless, and the element types used are TARGE169 and CONTA172. For boundary conditions, all lines in the axis $y=0$ are fixed in the $x$ direction, and the boundary at the bottom of the substrate is fixed in both the $x$ and the $y$ directions. The application of the load is displacement-controlled and was accomplished by assigning the corresponding deflection conditions to a pilot node virtually attached to the indenter geometry. Large deflections of the shell are accounted for in the analysis.

Three-dimensional analyses of hemispherical bubble shells are performed using ANSYS to understand the failure mechanism of the half-shells formed after the first crack occurred under compression. Three-dimensional analysis is needed due to the nonsymmetry of a hemispherical shell under compression in the equatorial plane, for which axisymmetry is not suitable. To reduce the simulation time, two planes of symmetry are applied to the three-dimensional geometry. A first plane of symmetry can be drawn by dividing the half-shell in two equal parts throughout the load axis, predicting a symmetrical deformation through that plane. A second plane of symmetry can be drawn by dividing the hemispherical shell in half through the perpendicular plane to the load axis, assuming the indenter is equal to the substrate, being rigid and with frictionless contact with the halfshell. Therefore, the three-dimensional analysis is performed in an eighth of a bubble shell where the inner area, the outer area, and one edge are free surfaces. The simulations of the hemispherical shell under compression assume an ideal initial state in which there are no deformations and the initial state of stress is null.

The element used for the 3D simulations is SOLID187, a 10-node element that has a quadratic displacement behavior, well-suited to modeling irregular meshes and with capability for large deflections and 
strains. The mesh has more than 30000 nodes and 19000 elements, with at least four elements radially spanning from the inner shell surface to the outer surface. The indenter is assumed to be rigid and the contact with the half-bubble shell is assumed to be frictionless. The element types used for the contact between indenter and substrate are TARGE170 and CONTA174. For boundary conditions, in addition to the symmetries applied, the top node of the shell is restricted in lateral directions $(x$ and $y)$ to avoid sliding of the shell during the simulation. The application of the load is displacement-controlled and was accomplished by assigning the corresponding failure displacement conditions to a pilot node virtually attached to the indenter geometry. The analysis used is nonlinear and allows for large deformations. Spherical coordinates are used for the extraction of the stress and strain components for a better understanding on the distribution of stresses and strains and possible failure causes.

The hemispherical calcined bubble shell is modeled as a perfect elastic material with two different Young's moduli of 13.7 and $20 \mathrm{GPa}$ and Poisson ratio of 0.18 . The hemispherical as-assembled bubble shell is modeled as a von Mises elastic-perfectly plastic material with Young's modulus of $6.9 \mathrm{GPa}$, yield stress of $0.12 \mathrm{GPa}$, and Poisson ratio of 0.18 . The geometric parameters, diameter, and thickness are defined to represent the average values experimentally measured and defined in Table 1 for uniform shells.

A Drucker-Prager material model is further used for the simulation of an as-assembled hemispherical shell. In this case, the element type used is SOLID65, a bricklike element defined by eight nodes having three degrees-of-freedom at each node: translations in the nodal $x, y$, and $z$ directions. A total of approximately 26000 elements are used for this simulation. An associated flow rule in conjunction with the Drucker-Prager yield function is adopted, which means that the yield and flow functions are identical.

Nanoparticle Film Generation and Characterization. For the generation of nanoparticle films, hydrophobic $\mathrm{SiO}_{2}$ nanoparticles (Nissan Chemical Industries, Ltd.) in toluene, at an approximate concentration of $14 \mathrm{wt} \%$, are deposited on a $\mathrm{Si}$ wafer (approximated size of $1.5 \times 1.5 \mathrm{~cm}^{2}$ ) by spin-coating using a WS-400BZ-6NPP/Lite spin-coater from Laurell Technologies Corp, at a rotation speed of $2000 \mathrm{rpm}$ for $2 \mathrm{~min}$. Prior to spin-coating, silicon wafers are treated with octadecyltrichlorosilane (OTS), which renders the substrates hydrophobic. To generate crack-free films that are thick enough for reliable nanoindentation, multideposition of nanoparticles is performed following a method previously reported. ${ }^{73}$ An intermediate step of dipping the film in DI water for a few minutes is necessary to allow for a better buildup of films during the multicoating steps. Three depositions are used to build a crack-free film of $450 \mathrm{~nm}$ in thickness. To facilitate a homogeneous buildup of crack-free films for calcined and sintered cases, the multideposition by spin-coating (3000 rpm for $2 \mathrm{~min}$ ) on OTS-treated quartz slides (approximated size of $1.5 \times 1.5$ $\mathrm{cm}^{2}$ ) is alternated with $2 \mathrm{~h}$ calcination steps at $700{ }^{\circ} \mathrm{C}$, which results in uniform films (thickness growth shown in Figure $\mathrm{S} 4$ of the Supporting Information). Five depositions are performed to obtain calcined films of $710 \mathrm{~nm}$, and these films are sintered at $1200{ }^{\circ} \mathrm{C}$ to obtain sintered films of $500 \mathrm{~nm}$. The verification of obtaining crack-free films is done by optical microscopy and SEM imaging. Film thickness and porosity of the films are determined using a J.A. Woolam $\alpha$-spectroscopic ellipsometer (SE) with a fixed incidence angle of $70^{\circ} .{ }^{74}$ The mechanical properties including Young's modulus and hardness of the as-assembled, calcined, and sintered films are obtained using nanoindentation performed using a Nano Indenter G200 from Agilent Technologies Inc. with continuous stiffness measurement using a Berkovich indenter tip following similar methods previously reported. ${ }^{75,76}$ The indenter tip area function is calibrated using fused silica, and a constant Young's modulus is achieved in the depth range of $40-100 \mathrm{~nm}$. The indenter is stabilized to achieve a thermal drift rate less than $0.05 \mathrm{~nm} / \mathrm{s}$ before performing any indentation. The indentation depths of the as-assembled, calcined, and sintered films are 200,300 , and $200 \mathrm{~nm}$, respectively, with a constant loading strain rate of $0.04 \mathrm{~s}^{-1}$. The characterization of the plasticity of as-assembled film and the determination of its yield strength are performed by measuring the hardness of the film using Berkovich and cube corner indenter tips following a previously reported method..77,78 The properties of the nanoparticle films are detailed in Table S1 of the Supporting Information.

Polymer-Bubble Composite Generation. Layer-by-layer (LbL) poymer-bubble composite is generated by first spin-coating a poly(vinyl alcohol) sacrificial layer on a glass slide (approximated size of $\left.2 \times 2 \mathrm{~cm}^{2}\right)$ using a $2 \mathrm{wt} \%$ PVA $(87-89 \%$ hydrolyzed, average MW $=13000-23000$, Sigma-Aldrich Co. LLC) in water solution at $2000 \mathrm{rpm}$. Subsequently, a polystyrene (PS, approx MW $=190$ 000, Scientific Polymer Products, Inc.) layer is spin-coated with a $20 \mathrm{wt} \%$ PS solution in toluene at $2000 \mathrm{rpm}$. Additional spin-coating steps with PS solution are performed to increase the thickness of the final PS layer up to the desired thickness. The film is dried at room conditions and is used to collect the bubbles using a Langmuir-Schaeffer technique. ${ }^{65}$ A monolayer of bubbles is then dried on top of the PS film. Subsequently, a new layer of PS is spin-coated on top of the bubbles covering the bubble monolayer. This process is repeated to reach the final desired composite thickness. The sacrificial PVA layer can be dissolved in water by immersing the film in water overnight, obtaining a free-standing LbL film of bubbles and polystyrene. Characterization of the fractured films is made by SEM.

\section{APPENDIX}

The Drucker-Prager yield criterion can be expressed as ${ }^{71}$

$$
\sigma_{\mathrm{VM}}+3 \beta \sigma_{\mathrm{m}}=\sigma_{\mathrm{Y}}
$$

where $\sigma_{\mathrm{VM}}$ is the pressure-independent von Mises effective stress $\left[\sigma_{\mathrm{VM}}{ }^{2}=(3 / 2) s_{i j} s_{i j}\right.$, where $s_{i j}=\sigma_{i j}-(1 / 3) \sigma_{k k} \delta_{i j}$ is the deviatoric stress] and $\sigma_{\mathrm{VM}}=(1 / 3) \sigma_{k k}$ is the mean stress. The material parameters entering the Drucker-Prager criterion are the pressure sensitivity $(\beta)$ and $\sigma_{\mathrm{Y}}$. Note that the yield stress in uniaxial tension is $\sigma_{\mathrm{T}}=\sigma_{\mathrm{Y}} /(1+\beta)$, and the yield stress in uniaxial compression is $\sigma_{\mathrm{C}}=\sigma_{\mathrm{Y}} /(1-\beta)$. For granular (frictional) materials, typically $\sigma_{\mathrm{C}}>\sigma_{\mathrm{T}}$, which implies $\beta>0$. From the overall load-displacement curves for the half-shells of as-assembled bubbles, i.e. after the first cracking event, the parameters chosen for the Drucker-Prager material are $\beta=$ 0.29 and $\sigma_{\mathrm{Y}}=83.6 \mathrm{MPa}$. Those parameters correspond to a compressive yield stress of $\sigma_{\mathrm{C}}=118 \mathrm{MPa}$, which agrees with the yield stress used for the von Mises model, and to a ratio of the compressive and tensile yield stress $\sigma_{\mathrm{C}} / \sigma_{\mathrm{T}}=1.82$ (also, the friction angle which is commonly used to characterize pressure sensitivity is $\left.22^{\circ}\right)$. An associated flow rule is adopted, ${ }^{72}$ which leads to plastic dilatancy.

\section{ASSOCIATED CONTENT}

\section{S Supporting Information}

I. TGA of as-assembled silica nanoparticle-shelled bubbles; II. Permeability of calcined bubbles; III. Determination of failure for nanoindentation tests; IV. Characterization of nanoparticle thin films; V. FEA results of sintered bubbles with different outer diameters, material properties and shell thicknesses; VI. Differences between ex situ nanoindentation and in situ compression mechanical testing; VII. In situ compression test movies; VIII. Three-dimensional FEA of half-shells for asassembled and calcined bubbles. This material is available free of charge via the Internet at http://pubs.acs.org

\section{AUTHOR INFORMATION}

\section{Corresponding Authors}

*D.S.G. e-mail: gianola@seas.upenn.edu.

*J.L.B. e-mail: bassani@seas.upenn.edu.

*G.F. e-mail: gang.feng@villanova.edu.

*D.L. e-mail: daeyeon@seas.upenn.edu 


\section{Author Contributions}

The manuscript was written through contributions of all authors. All authors have given approval to the final version of the manuscript.

\section{Notes}

The authors declare no competing financial interest.

\section{ACKNOWLEDGMENTS}

This work is supported by the National Science Foundation through PENN MRSEC DMR-1120901 and DMR-1055594. T.B. acknowledges the graduate fellowship from Obra Social "La Caixa". D.L. acknowledges support from the 3M Nontenured Faculty Award. Hydrophobic silica nanoparticles used in this study were generously provided by Nissan Chemical Industries, Ltd. (Japan). J.L.B. acknowledges financial support from the National Science Foundation CMMI 09-00058.

\section{REFERENCES}

(1) Rohatgi, P. K.; Gupta, N.; Weiss, D.; Miracle, D. Synthesis and Applications of Cast Metal Matrix Composites and Syntactic Foams. In SAMPE Fall Technical Conference Global Advances in Materials and Process Engineering: 38th International SAMPE Technical Conference, Dallas, TX, Nov 6-9, 2006; Society for the Advancement of Material and Process Engineering: Covina, CA, 2006.

(2) Peter, S. L.; Mylavarapu, P.; Woldesenbet, E. High Strain Rate Properties of Nanoparticulate Syntactic Foams. In 22nd Technical Conference of the American Society for Composites 2007, Seattle, WA, Sept 17-19, 2007; Curran Associates, Inc.: Red Hook, NY, 2007; pp 1916-1926.

(3) Konka, H. P.; Wahab, M. A.; Lian, K. Sandwich Structures with Smart Composite Face Skin. In ASME 2011 International Mechanical Engineering Congress and Exposition; Denver, CO, Nov 11-17, 2011; ASME: New York, 2011; pp 157-166.

(4) Conover, D.; Nikolaus, R. Innovation and Unity in Advancing Ocean Sciences. Sea Technol. 2013, 54 (1), 19-20.

(5) Fleck, N. A.; Deshpande, V. S.; Ashby, M. F. Micro-Architectured Materials: Past, Present and Future. Proc. R. Soc. London, Ser. A 2010, 466 (2121), 2495-2516.

(6) Ashby, M. F.; Gibson, L. J.; Wegst, U.; Olive, R. The Mechanical Properties of Natural Materials. I. Material Property Charts. Proc. $R$. Soc. London, Ser. A 1995, 450 (1938), 123-140.

(7) Wegst, U. G. K.; Ashby, M. F. The Mechanical Efficiency of Natural Materials. Philos. Mag. 2004, 84 (21), 2167-2181.

(8) Jang, D.; Meza, L. R.; Greer, F.; Greer, J. R. Fabrication and Deformation of Three-Dimensional Hollow Ceramic Nanostructures. Nat. Mater. 2013, 12 (10), 893-898.

(9) Patankar, S. N.; Kranov, Y. A. Hollow Glass Microsphere HDPE Composites for Low Energy Sustainability. Mater. Sci. Eng., A 2010, 527 (6), 1361-1366.

(10) Bubbles in this work refer to stable hollow particles that can be further processed for the generation of composites.

(11) Fiedler, T.; Richards, H. S.; Belova, I. V.; Ochsner, A.; Murch, G. E. Experimental Analysis on the Thermal Anisotropy of Syntactic Hollow Sphere Structures. Exp. Therm. Fluid Sci. 2013, 44 (0), 637641.

(12) Grosjean, F.; Bouchonneau, N.; Choqueuse, D.; SauvantMoynot, V. Comprehensive Analyses of Syntactic Foam Behaviour in Deepwater Environment. J. Mater. Sci. 2009, 44 (6), 1462-1468.

(13) Lee, J.-H.; Singer, J. P.; Thomas, E. L. Micro-/Nanostructured Mechanical Metamaterials. Adv. Mater. 2012, 24 (36), 4782-4810.

(14) Dombrovsky, L. A.; Randrianalisoa, J. H.; Baillis, D. Infrared Radiative Properties of Polymer Coatings Containing Hollow Microspheres. Int. J. Heat Mass Transfer 2007, 50 (7-8), 1516-1527.

(15) Shunmugasamy, V. C.; Pinisetty, D.; Gupta, N. Thermal Expansion Behavior of Hollow Glass Particle/Vinyl Ester Composites. J. Mater. Sci. 2012, 47 (14), 5596-5604.
(16) Colombo, P.; Degischer, H. P. Highly Porous Metals and Ceramics. Mater. Sci. Technol. 2010, 26 (10), 1145-1158.

(17) Bunn, P.; Mottram, J. T. Manufacture and Compression Properties of Syntactic Foams. Composites 1993, 24 (7), 565-571.

(18) Gupta, N.; Kishore; Woldesenbet, E.; Sankaran, S. Studies on Compressive Failure Features in Syntactic Foam Material. J. Mater. Sci. 2001, 36 (18), 4485-4491.

(19) Gupta, N. A Functionally Graded Syntactic Foam Material for High Energy Absorption under Compression. Mater. Lett. 2007, 61 (4-5), 979-982.

(20) Kallas, D. H.; Chatten, C. K. Buoyancy Materials for Deep Submergence. Ocean Eng. 1969, 1 (4), 421-\&.

(21) Chen, Z.; Xu, C.; Ma, C.; Ren, W.; Cheng, H. M. Lightweight and Flexible Graphene Foam Composites for High-Performance Electromagnetic Interference Shielding. Adv. Mater. 2013, 25 (9), $1296-300$.

(22) Göransson, P. Advanced Materials and Structures for Noise Control. In 6th European Conference on Noise Control: Advanced Solutions for Noise Control, Tampere, Finland, May 30-June 1, 2006.

(23) Tochizawa, M.; Tanaka, M. Development of Lightweight Single Bumper Shield. In 54th International Astronautical Congress of the International Astronautical Federation (IAF), the International Academy of Astronautics and the International Institute of Space Law; Bremen, Germany, Sept 29-Oct 3, 2003; Curran Associates, Inc.: Red Hook, NY, 2003; pp 3629-3635.

(24) Dobrounig, O. Football. US Patent 6991569-B2, Jan 31, 2006.

(25) d'Almeida, J. R. M. An Analysis of the Effect of the Diameters of Glass Microspheres on the Mechanical Behavior of Glass-Microsphere/Epoxy-Matrix Composites. Compos. Sci. Technol. 1999, 59 (14), 2087-2091.

(26) Thomas, C. R. Syntactic Carbon Foams. Mater. Sci. Eng. 1973, 12 (5-6), 219-233.

(27) Kyung H. Moh, S. P. M. N.; Harold G. Sowman, S. P. M. N.; Thomas E. Wood, S. P. M. N. Sol Gel-Derived Ceramic Bubbles. US Patent 5077241, 1991.

(28) Garza-Cruz, T. V.; Nakagawa, M. On a Hybrid Method to Characterize the Mechanical Behavior of Thin Hollow Glass Microspheres. Granular Matter 2012, 14 (3), 309-318.

(29) Koopman, M.; Gouadec, G.; Carlisle, K.; Chawla, K. K.; Gladysz, G. Compression Testing of Hollow Microspheres (Microballoons) To Obtain Mechanical Properties. Scr. Mater. 2004, 50 (5), 593-596.

(30) Gladysz, G. M.; Perry, B.; Mceachen, G.; Lula, J. Three-Phase Syntactic Foams: Structure-Property Relationships. J. Mater. Sci. 2006, 41 (13), 4085-4092.

(31) Lee, M. H.; Prasad, V.; Lee, D. Microfluidic Fabrication of Stable Nanoparticle-Shelled Bubbles. Langmuir 2010, 26 (4), 222730.

(32) Brugarolas, T.; Park, B. J.; Lee, M. H.; Lee, D. Generation of Amphiphilic Janus Bubbles and Their Behavior at an Air-Water Interface. Adv. Funct. Mater. 2011, 21 (20), 3924-3931.

(33) Brugarolas, T.; Tu, F. Q.; Lee, D. Directed Assembly of Particles Using Microfluidic Droplets and Bubbles. Soft Matter 2013, 9 (38), 9046-9058.

(34) Lee, M. H.; Lee, D. Elastic Instability of Polymer-Shelled Bubbles Formed from Air-in-Oil-in-Water Compound Bubbles. Soft Matter 2010, 6 (18), 4326-4330.

(35) Silpe, J. E.; Nunes, J. K.; Poortinga, A. T.; Stone, H. A. Generation of Antibubbles from Core-Shell Double Emulsion Templates Produced by Microfluidics. Langmuir 2013, 29 (28), 8782-7.

(36) Kotula, A. P.; Anna, S. L. Probing Timescales for Colloidal Particle Adsorption Using Slug Bubbles in Rectangular Microchannels. Soft Matter 2012, 8 (41), 10759-10772.

(37) Duncanson, W. J.; Abbaspourrad, A.; Shum, H. C.; Kim, S. H.; Adams, L. L.; Weitz, D. A. Monodisperse Gas-Filled Microparticles from Reactions in Double Emulsions. Langmuir 2012, 28 (17), 67425. 
(38) Chen, H. S.; Li, J.; Wan, J. D.; Weitz, D. A.; Stone, H. A. GasCore Triple Emulsions for Ultrasound Triggered Release. Soft Matter 2013, 9 (1), 38-42.

(39) Park, J. I.; Nie, Z.; Kumachev, A.; Abdelrahman, A. I.; Binks, B. P.; Stone, H. A.; Kumacheva, E. A Microfluidic Approach to Chemically Driven Assembly of Colloidal Particles at Gas-Liquid Interfaces. Angew. Chem., Int. Ed. 2009, 48 (29), 5300-4.

(40) Tumarkin, E.; Park, J. I.; Nie, Z.; Kumacheva, E. Temperature Mediated Generation of Armoured Bubbles. Chem. Commun. 2011, 47 (47), 12712-4.

(41) Yang, L.; Wang, K.; Mak, S.; Li, Y.; Luo, G. A Novel Microfluidic Technology for the Preparation of Gas-in-Oil-in-Water Emulsions. Lab Chip 2013, 13 (17), 3355-9.

(42) Wang, W. T.; Chen, R.; Xu, J. H.; Wang, Y. D.; Luo, G. S. OneStep Microfluidic Approach for Controllable Production of Gas-inWater-in-Oil (G/W/O) Double Emulsions and Hollow Hydrogel Microspheres. RSC Adv. 2014, 4 (32), 16444-16448.

(43) Xu, J. H.; Chen, R.; Wang, Y. D.; Luo, G. S. Controllable Gas/ Liquid/Liquid Double Emulsions in a Dual-Coaxial Microfluidic Device. Lab Chip 2012, 12 (11), 2029-36.

(44) Chen, R.; Dong, P. F.; Xu, J. H.; Wang, Y. D.; Luo, G. S. Controllable Microfluidic Production of Gas-in-Oil-in-Water Emulsions for Hollow Microspheres with Thin Polymer Shells. Lab Chip 2012, 12 (20), 3858-60.

(45) Lankveld, J. M. G.; Lyklema, J. Adsorption of Polyvinyl Alcohol on the Paraffin-Water Interface. I. Interfacial Tension as a Function of Time and Concentration. J. Colloid Interface Sci. 1972, 41 (3), 454465.

(46) Wu, Z.; Lee, D.; Rubner, M. F.; Cohen, R. E. Structural Color in Porous, Superhydrophilic, and Self-Cleaning $\mathrm{SiO}_{2} / \mathrm{TiO}_{2}$ Bragg Stacks. Small 2007, 3 (8), 1445-51.

(47) Choi, S. Y.; Mamak, M.; von Freymann, G.; Chopra, N.; Ozin, G. A. Mesoporous Bragg Stack Color Tunable Sensors. Nano Lett. 2006, 6 (11), 2456-61.

(48) Yaghoubi, H.; Taghavinia, N.; Alamdari, E. K.; Volinsky, A. A. Nanomechanical Properties of $\mathrm{TiO}_{2}$ Granular Thin Films. ACS Appl. Mater. Interfaces 2010, 2 (9), 2629-36.

(49) Peng, Z.; Kong, L. X. A Thermal Degradation Mechanism of Polyvinyl Alcohol/Silica Nanocomposites. Polym. Degrad. Stab. 2007, 92 (6), 1061-1071.

(50) Caruso, F.; Caruso, R. A.; Mohwald, H. Nanoengineering of Inorganic and Hybrid Hollow Spheres by Colloidal Templating. Science 1998, 282 (5391), 1111-4.

(51) O’Hayre, R.; Feng, G.; Nix, W. D.; Prinz, F. B. Quantitative Impedance Measurement Using Atomic Force Microscopy. J. Appl. Phys. 2004, 96 (6), 3540-3549.

(52) Pauchard, L.; Rica, S. Contact and Compression of Elastic Spherical Shells: The Physics of a 'Ping-Pong' Ball. Philos. Mag. B 1998, 78 (2), 225-233.

(53) Carlisle, K. B.; Lewis, M.; Chawla, K. K.; Koopman, M.; Gladysz, G. M. Finite Element Modeling of the Uniaxial Compression Behavior of Carbon Microballoons. Acta Mater. 2007, 55 (7), 2301-2318.

(54) Lee, D.; Jia, S.; Banerjee, S.; Bevk, J.; Herman, I. P.; Kysar, J. W. Viscoplastic and Granular Behavior in Films of Colloidal Nanocrystals. Phys. Rev. Lett. 2007, 98 (2), 026103.

(55) Bassani, J. L. Linear Densification and Microcracking in Sintering Compacts. Mech. Mater. 1991, 12 (2), 119-130.

(56) Simulations for uniform shells with different outer diameters (and the different Young's moduli), representing the standard deviation measured experimentally, are performed using FEA. FEA results (see Figure S5, Supporting Information) confirm that the change in the diameter (and Young's moduli) does not significantly affect the mechanical response of the shells.

(57) Datta, S. S.; Kim, S. H.; Paulose, J.; Abbaspourrad, A.; Nelson, D. R; Weitz, D. A. Delayed Buckling and Guided Folding of Inhomogeneous Capsules. Phys. Rev. Lett. 2012, 109 (13), 134302.

(58) Paulose, J.; Nelson, D. R. Buckling Pathways in Spherical Shells with Soft Spots. Soft Matter 2013, 9 (34), 8227-8245.
(59) Gianola, D. S.; Sedlmayr, A.; Monig, R.; Volkert, C. A.; Major, R. C.; Cyrankowski, E.; Asif, S. A.; Warren, O. L.; Kraft, O. In Situ Nanomechanical Testing in Focused Ion Beam and Scanning Electron Microscopes. Rev. Sci. Instrum. 2011, 82 (6), 063901.

(60) Magagnosc, D. J.; Ehrbar, R.; Kumar, G.; He, M. R.; Schroers, J.; Gianola, D. S., Tunable Tensile Ductility in Metallic Glasses. Sci. Rep. 2013, 3.

(61) Carlisle, K. B.; Koopman, M.; Chawla, K. K.; Kulkarni, R.; Gladysz, G. M.; Lewis, M. Microstructure and Compressive Properties of Carbon Microballoons. J. Mater. Sci. 2006, 41 (13), 3987-3997.

(62) Gupta, N.; Woldesenbet, E.; Mensah, P. Compression Properties of Syntactic Foams: Effect of Cenosphere Radius Ratio and Specimen Aspect Ratio. Composites, Part A 2004, 35 (1), 103111.

(63) Drucker, D. C.; Prager, W. Soil Mechanics and Plastic Analysis or Limit Design. Q. Appl. Math. 1952, 10 (2), 157-165.

(64) Koopman, M.; Chawla, K. K.; Carlisle, K. B.; Gladysz, G. M. Microstructural Failure Modes in Three-Phase Glass Syntactic Foams. J. Mater. Sci. 2006, 41 (13), 4009-4014.

(65) Santhanam, V.; Andres, R. P. Microcontact Printing of Uniform Nanoparticle Arrays. Nano Lett. 2004, 4 (1), 41-44.

(66) Bardella, L.; Genna, F. On the Elastic Behavior of Syntactic Foams. Int. J. Solids Struct. 2001, 38 (40-41), 7235-7260.

(67) Chen, Z.; Yan, Q.; Huang, Z.; Shen, Q.; Zhang, L. Preparation and Characterization of Novel Functional Gradient Syntactic Foam. Adv. Mater. Res. 2009, 66, 284-287.

(68) Glikman, Z.; Altus, E. Strain Measurement in Syntactic Foam Using an Embedded Fiber-Optic Sensor. In Advances and Trends in Engineering Materials and Their Applications; AES-ATEMA 1st International Conference; Haddad, Y. M., Ed.; AES-Advanced Engineering Solutions: Ottawa, Canada, 2007; pp 475-483.

(69) Xu, W.; Li, G. Constitutive Modeling of Shape Memory Polymer Based Self-Healing Syntactic Foam. Int. J. Solids Struct. 2010, 47 (9), 1306-1316.

(70) Xue, X. B.; Zhao, Y. Y.; Kearns, V.; Williams, R. L. Mechanical and Biological Properties of Titanium Syntactic Foams. In Supplemental Proceedings: Volume 2: Material Characterization, Computation, Modeling, and Energy TMS; The Minerals, Metals \& Materials Society: Warrendale, PA, 2010; pp 129-135.

(71) Alejano, L. R.; Bobet, A. Drucker-Prager Criterion. Rock Mech. Rock Eng. 2012, 45 (6), 995-999.

(72) Lubliner, J., Yield Criteria, Flow Rules, and Hardening Rules. In Plasticity Theory; Macmillan Publishing Co.: New York, 1990; Chapter 3, pp 125-136.

(73) Prosser, J. H.; Brugarolas, T.; Lee, S.; Nolte, A. J.; Lee, D. Avoiding Cracks in Nanoparticle Films. Nano Lett. 2012, 12 (10), 5287-91.

(74) Lee, D.; Rubner, M. F.; Cohen, R. E. All-Nanoparticle ThinFilm Coatings. Nano Lett. 2006, 6 (10), 2305-12.

(75) Zhang, L.; Prosser, J. H.; Feng, G.; Lee, D. Mechanical Properties of Atomic Layer Deposition-Reinforced Nanoparticle Thin Films. Nanoscale 2012, 4 (20), 6543-52.

(76) Zhang, L.; Feng, G.; Zeravcic, Z.; Brugarolas, T.; Liu, A. J.; Lee, D. Using Shape Anisotropy To Toughen Disordered Nanoparticle Assemblies. ACS Nano 2013, 7 (9), 8043-8050.

(77) Feng, G.; Qu, S.; Huang, Y.; Nix, W. D. An Analytical Expression for the Stress Field around an Elastoplastic Indentation/ Contact. Acta Mater. 2007, 55 (9), 2929-2938.

(78) Bucaille, J. L.; Stauss, S.; Felder, E.; Michler, J. Determination of Plastic Properties of Metals by Instrumented Indentation Using Different Sharp Indenters. Acta Mater. 2003, 51 (6), 1663-1678. 